\title{
Mapping Land Cover Through Time with the Rapid Land Cover Mapper-Documentation and User Manual
}

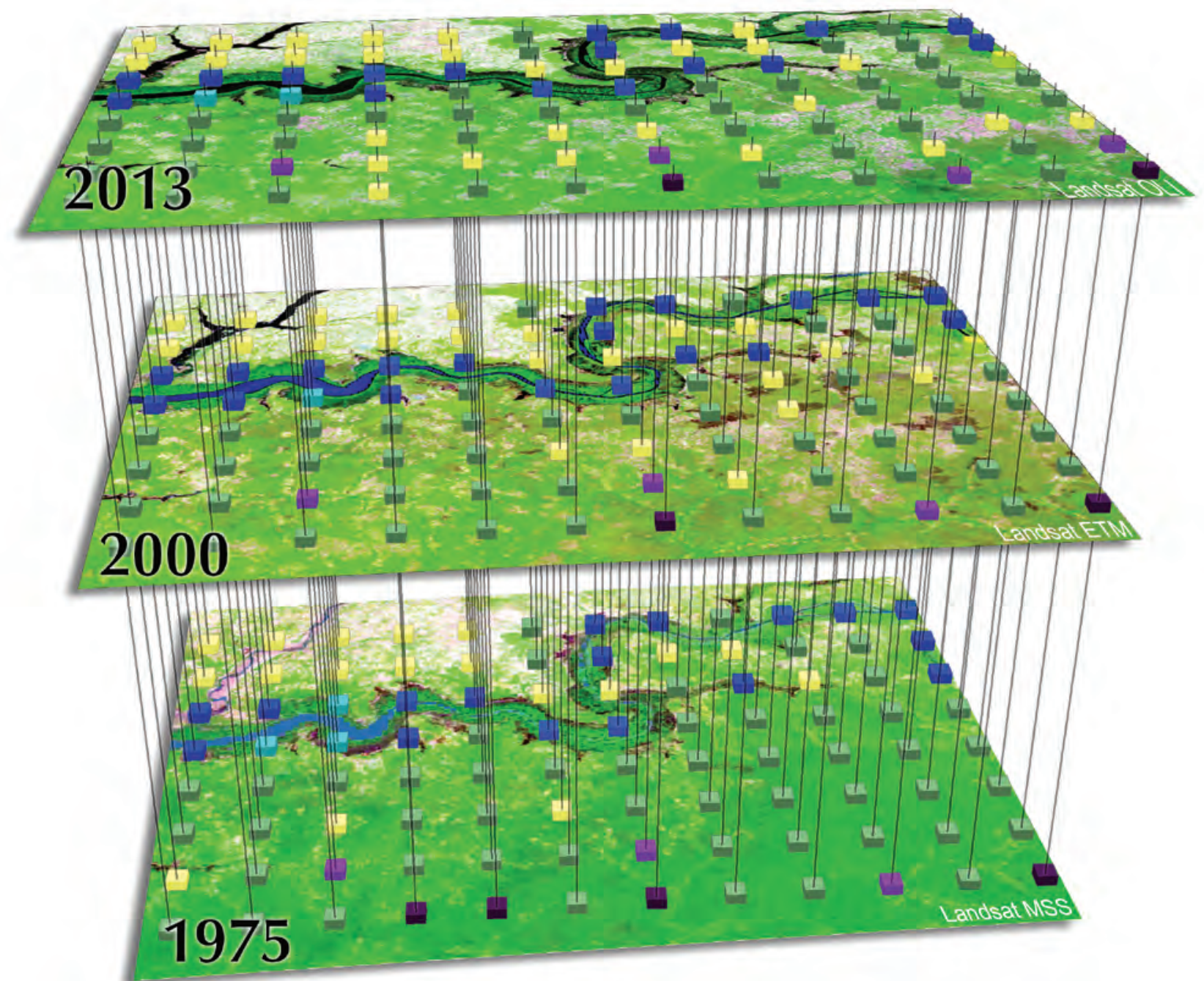

Open-File Report 2017-1012 



\section{Mapping Land Cover Through Time with the Rapid Land Cover Mapper-Documentation and User Manual}

By Suzanne E. Cotillon and Melissa L. Mathis

Open-File Report 2017-1012 


\title{
U.S. Geological Survey William H. Werkheiser, Acting Director
}

\author{
U.S. Geological Survey, Reston, Virginia: 2017
}

For more information on the USGS - the Federal source for science about the Earth, its natural and living resources, natural hazards, and the environment-visit https://www.usgs.gov or call 1-888-ASK-USGS.

For an overview of USGS information products, including maps, imagery, and publications, visit https://store.usgs.gov/.

Any use of trade, firm, or product names is for descriptive purposes only and does not imply endorsement by the U.S. Government.

Although this information product, for the most part, is in the public domain, it also may contain copyrighted materials as noted in the text. Permission to reproduce copyrighted items must be secured from the copyright owner.

Suggested citation:

Cotillon, S.E., and Mathis, M.L., 2017, Mapping land cover through time with the Rapid Land Cover Mapper-Documentation and user manual: U.S. Geological Survey Open File Report 2017-1012, 23 p., https://doi.org/10.3133/ ofr20171012.

ISSN 2331-1258 (online) 


\section{Contents}

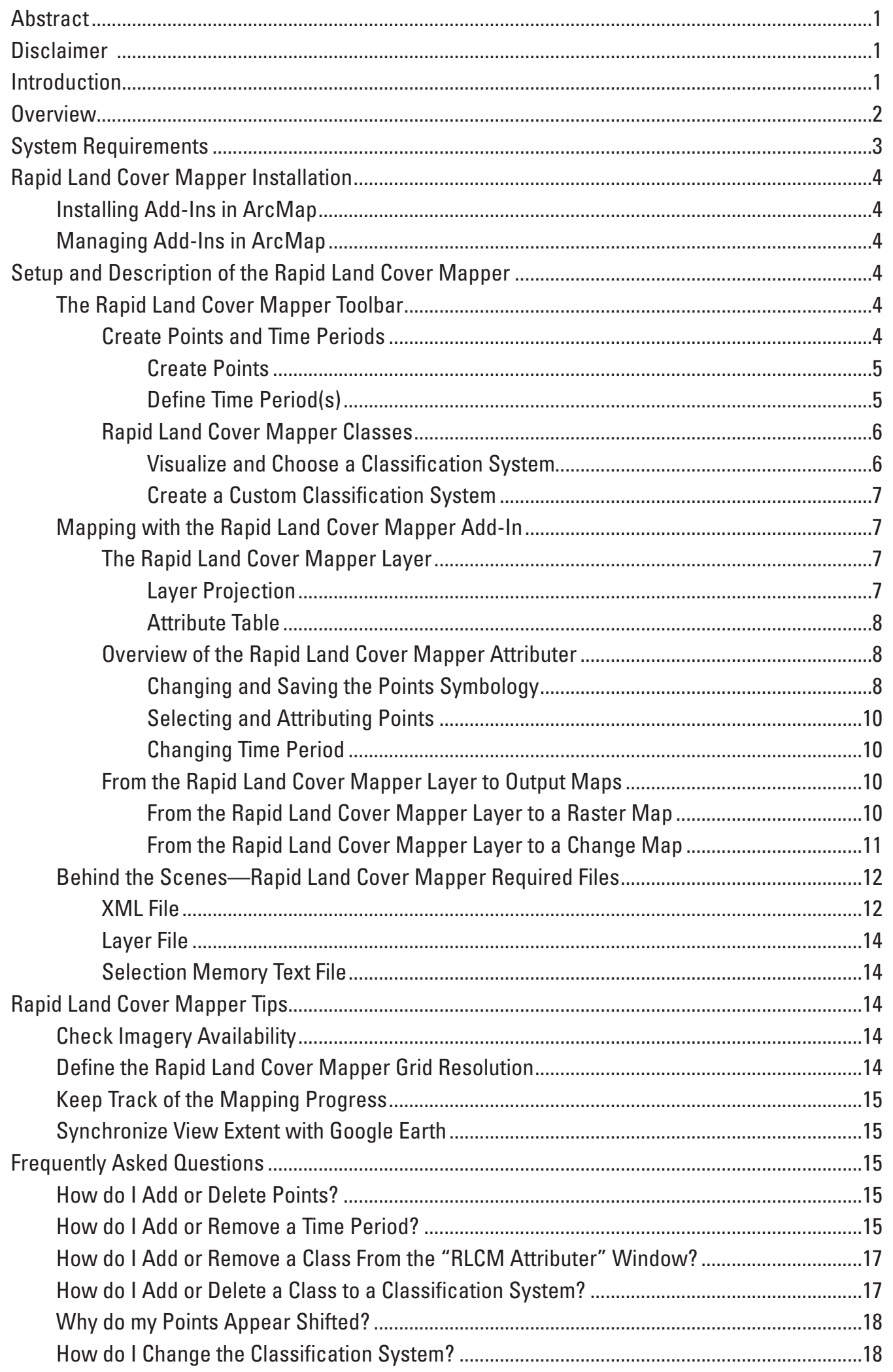


Frequently Asked Questions-Continued

I Have Added a Class but it is Not Showing Up in the "RLCM Attributer" Window or in

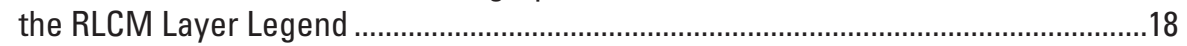

How do I Cancel if I Made a Mistake Attributing My Points? .................................................18

Can I Change the Resolution of My Map After I Have Started Mapping? ..............................18

Can I Have Two Rapid Land Cover Mapper Layers in One ArcMap Project?..........................18

Can I Change the Projection of the Rapid Land Cover Mapper Layer? ..................................18

Why is the Tool Slow When Attributing and Selecting Points? .............................................18

Can I Create Dots at a Scale Less Than 1:800,000? ...........................................................

Why Can I Not Create Points Within the Mask? ................................................................

I Get an Error Message When Trying to Open the "RLCM Attributer" Window. How do

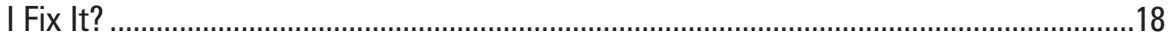

When Using the "Google Earth" Button, Why do the Google Earth Images Appear

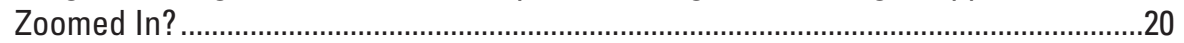

What do I do if the Rapid Land Cover Mapper Add-In Keeps Crashing? ...............................20

What do I do if I Get an Error Message When I Try to Create Points? ...................................20

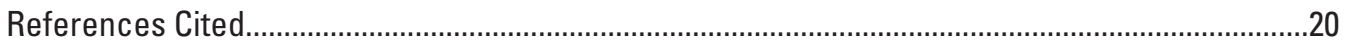

Appendix 1. Classification Systems Included in the Rapid Land Cover Mapper Add-In.............22

Appendix 2. List of the Rapid Land Cover Mapper Projection Files ............................................22

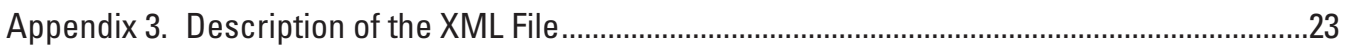

\section{Figures}

1-15. Screen shots showing:

1. Steps in making a land use and land cover map using the Rapid Land Cover Mapper add-in.

2. A simplified time-series representation of three land use and land cover interpretations using the Rapid Land Cover Mapper add-in ......................................

3. The "RLCM Toolbar" buttons and options ..............................................................

4. The "RLCM Layer and Time Periods" window.....................................................5

5. Add a time period from the "RLCM Layer and Time Periods" window .....................6

6. The "RLCM Classification System" window ...........................................................

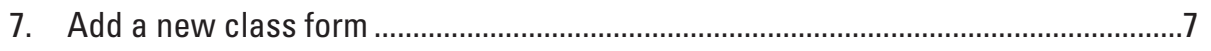

8. Example of attributed points in the RLCM layer attribute table for one time

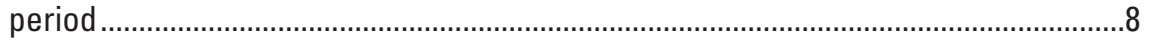

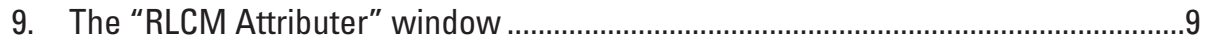

10. Examples of default and custom symbologies for several land cover classes ........9

11. Selecting and attributing points with common land cover class .............................10

12. Copy attributes from one time period to another ...................................................11

13. Process for quick multiple-time period mapping using the Rapid Land Cover Mapper

14. The "Data Analysis" drop-down menu ..............................................................12

15. The "Create Raster from Time Periods" window ...................................................12 
16-23. Screen shots showing:

16. Example of the conversion of a Rapid Land Cover Mapper point layer to a

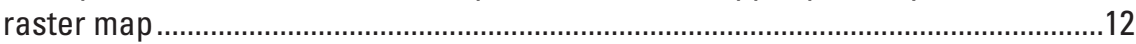

17. Example of the attribute table of a Rapid Land Cover Mapper raster for the year 2000

18. The "RLCM Change Map" window.......................................................................13

19. Example of a change raster and associated attribute table .................................14

20. Tip to keep track of the multiple time period's mapping progress using the example of land cover mapping in Benin ..............................................................15

21. Time period record in the .xml file of the Rapid Land Cover Mapper layer .............17

22. "Coordinate System" tab of the "Data Frame Properties" window .........................19

23. Shifted points and aligned points after updating the data frame projection...........20

\section{Appendix Figures}

2-1. Diagram showing projections embedded in the Rapid Land Cover Mapper add-in .......22

3-1. Screen shot showing the structure of an .xml file from a Rapid Land Cover Mapper layer.Shifted points and aligned points after updating the data frame projection ..........23

\section{Table}

1. Suggested imagery source and number of points in the point grid based on points resolution and study area size

\section{Appendix Table}

1-1. Description of the classification systems embedded in the Rapid Land Cover Mapper add-in 


\section{Conversion Factors}

International System of Units to U.S. customary units

\begin{tabular}{lcl}
\hline \multicolumn{1}{c}{ Multiply } & By & \multicolumn{1}{c}{ To obtain } \\
\hline meter $(\mathrm{m})$ & Length & \\
meter $(\mathrm{m})$ & 3.281 & foot $(\mathrm{ft})$ \\
kilometer $(\mathrm{km})$ & 1.094 & yard (yd) \\
\hline & 0.6214 & mile (mi) \\
\hline square kilometer $\left(\mathrm{km}^{2}\right)$ & Area & acre \\
square kilometer $\left(\mathrm{km}^{2}\right)$ & 247.1 & square mile $\left(\mathrm{mi}^{2}\right)$ \\
\hline
\end{tabular}

\section{Abbreviations}

RLCM Rapid Land Cover Mapper

USGS U.S. Geological Survey 


\title{
Mapping Land Cover Through Time with the Rapid Land Cover Mapper-Documentation and User Manual
}

\author{
By Suzanne E. Cotillon ${ }^{1}$ and Melissa L. Mathis ${ }^{1}$
}

\begin{abstract}
The Rapid Land Cover Mapper is an Esri ArcGIS ${ }^{\circledR}$ Desktop add-in, which was created as an alternative to automated or semiautomated mapping methods. Based on a manual photo interpretation technique, the tool facilitates mapping over large areas and through time, and produces time-series raster maps and associated statistics that characterize the changing landscapes. The Rapid Land Cover Mapper add-in can be used with any imagery source to map various themes (for instance, land cover, soils, or forest) at any chosen mapping resolution. The user manual contains all essential information for the user to make full use of the Rapid Land Cover Mapper add-in. This manual includes a description of the add-in functions and capabilities, and step-by-step procedures for using the add-in. The Rapid Land Cover Mapper add-in was successfully used by the U.S. Geological Survey West Africa Land Use Dynamics team to accurately map land use and land cover in 17 West African countries through time (1975, 2000, and 2013).
\end{abstract}

\section{Disclaimer}

This software has been approved for release by the U.S. Geological Survey (USGS). Although the software has been subjected to rigorous review, the USGS reserves the right to update the software as needed pursuant to further analysis and review. No warranty, expressed or implied, is made by the USGS or the U.S. Government as to the functionality of the software and related material, nor shall the fact of release constitute any such warranty; furthermore, the software is released on condition that neither the USGS nor the U.S. Government shall be held liable for any damages resulting from its authorized or unauthorized use.

${ }^{1}$ Suzanne E. Cotillon, a contractor: SGT Inc.; Work done under contract to U.S. Geological Survey.

${ }^{1}$ Melissa L. Mathis, a contractor: SGT Inc.; Work done under contract to U.S. Geological Survey.

\section{Introduction}

Mapping land use and land cover over large areas and through time has always presented substantial challenges. Mapping land cover through time requires an approach that generates consistently accurate maps for reliable change detection. Two contrasting approaches are used to map land cover: automated classification and visual image interpretation. The first and most common approach is to compute automated classification based on the image pixel value - this method involves inputting digital image data into an image classifier. Spectral reflectance of land surfaces - and more broadly spectral response patterns - measured by remote sensors may be quantitative but is not absolute; also, the spectral reflectance may be distinctive but is not necessarily unique to each land cover type. Spectral reflectance associated with land cover type is extremely variable, which often can lead to misclassification (Lillesand and others, 2015). This variability poses substantial challenges in mapping and analyzing land cover types based solely on their spectral reflectance. Although the automated method is fast and efficient in classifying large areas, accuracy often is lacking in the output maps when comparing two or more time periods.

Mapping land use and land cover using visual interpretation is not without its own challenges, but the combination of firsthand knowledge of the landscapes and reliance on multiple dimensions of information inherent in imagery is a powerful approach to producing highly accurate maps. The manual photo interpretation method produces good results for timeseries mapping but is labor-intensive over large areas. The Rapid Land Cover Mapper (RLCM) add-in was developed to address this challenge.

The RLCM add-in is a vector and raster hybrid approach that lends itself to multiple-resolution and time-series mapping. Conceptually, the add-in is based on the traditional point grid method for calculating areas that has long been used by foresters and other aerial photography users (Schumaher and Chapman, 1942). The RLCM add-in generates a grid of digital points that overlays on an array of imagery displayed in ArcGIS ${ }^{\circledR}$. Using image interpretation techniques, the analyst 
identifies a discrete land use and land cover class for each point. Once the analyst classifies all points for a given time period, a raster map can be generated through the RLCM addin for further analysis. The same process can be applied to the same area for different time periods, and the resulting data can be compared to assess change (Comité Permanent Inter-états de Lutte contre la Sécheresse dans le Sahel [CILSS], 2016).

The RLCM approach has substantial advantages. First, as in photo interpretation, the RLCM add-in enables an analyst to compare images from different sources and of varied scales and formats. Second, based on their experience in visual interpretation and their knowledge of the field, interpreters can assess landscape characteristics (vegetation, soils, geology, human use of the land, and so forth). Third, at an optimal sampling resolution this method is efficient for large area mapping. Fourth, because the RLCM approach is based on point-grid sampling, and the same location is sampled through time, this tool is optimal for time-series mapping. Finally, the RLCM add-in allows the use of nested point-grid sampling, creating multiple-resolution datasets. This nesting technique enables the user to complete a more detailed analysis of a study area (see appendix 1).

\section{Overview}

The RLCM add-in was developed to be used in Esri's ArcGIS Desktop (http://desktop.arcgis.com/) environment by advanced Geographic Information System users. Although the tool was designed for land use and land cover mapping, any set of features, such as soil, geology, urban features, or any other themes, that are discernible in the imagery can be classified. The RLCM add-in allows the user to select from seven predefined land cover classification systems or create a custom classification. Before using the add-in, the user needs to define the geographic extent of the study area, choose the mapping resolution, choose the time periods (years) to analyze, and acquire imagery for all selected time periods. Once these steps are completed, the user can use the RLCM add-in to generate a digital grid of points that overlays an image and begin the classification process using standard photo interpretation techniques (fig. 1). The analyst identifies the discrete land use and land cover class for each point. The RLCM add-in facilitates the selection and assignment of the point's land cover class, which is possible because of simultaneous point selection and cascading period classification. Simultaneous selection allows

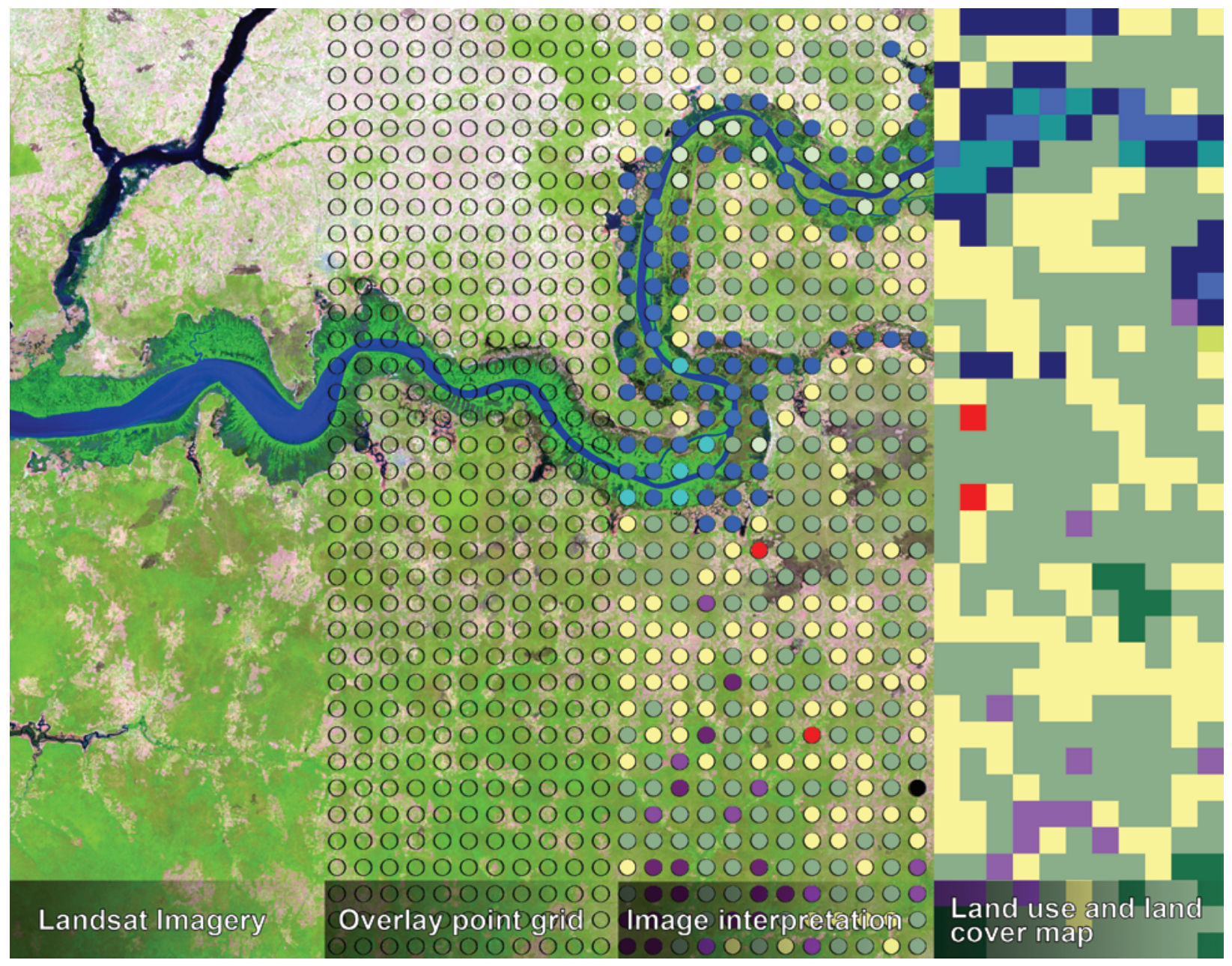

Figure 1. Steps in making a land use and land cover map using the Rapid Land Cover Mapper add-in (from 30-meter resolution Landsat imagery to 2-kilometer resolution land cover raster). 
the analyst to select many points of a common class and assign them to that specific class with one action.

Cascading is a method of completing the classification of the first time period for a given area, then pushing that classification information forward or back in time (fig. 2). After copying the attributed points into another time period, they are displayed over images that correspond to the "new" time period. The interpretation process is repeated, and in this case the previously attributed points are reviewed to determine if they should remain unchanged or be edited to reflect a change in the land cover. Generally, the image analysts begin with the most recent time period, then work back in time to the earlier time periods. This results in the production of multiperiod land use and land cover maps and associated statistics that characterize the changing landscapes at national and regional scales.

\section{System Requirements}

ArcGIS Desktop 10.2 or higher is required to use the RLCM add-in. Individual RLCM add-ins are available for each version. The add-in was created in Visual Basic .NET using Microsoft巴 Visual Studio (https://www.visualstudio. $\mathrm{com} /$ ) as a custom add-in for ArcMap ${ }^{\mathrm{TM}}$ (http://desktop.arcgis. com/en/arcmap/), and it was developed and tested on systems running Microsoft Windows 7. The add-in should function with later versions of the Windows operating system supported by ArcGIS Desktop 10.x. The RLCM add-in can synchronize ArcMap's window extent with Google Earth (https://www. google.com/earth/). Although not required, Google Earth is recommended to use the add-in's features.

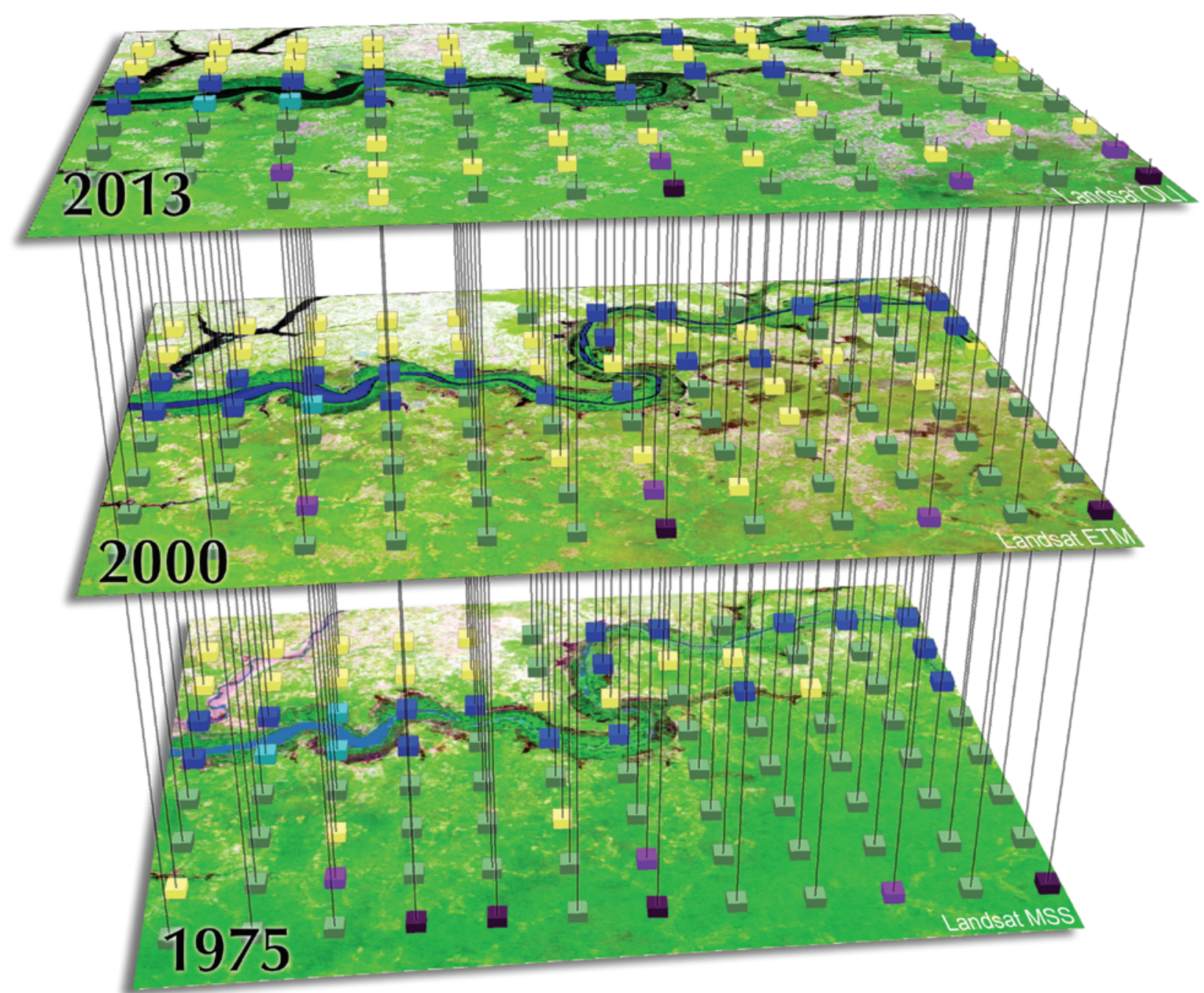

Figure 2. A simplified time-series representation of three land use and land cover interpretations using the Rapid Land Cover Mapper add-in. The cubes symbolize the grid points that are placed over the imagery, color coded by land cover class. The vertical lines show the spatial alignment of the points through time. 


\section{Rapid Land Cover Mapper Installation}

The RLCM is an ArcGIS add-in that provides custom functionality to ArcMap. Before installing the add-in, close all open ArcMap applications. Make sure to download the RLCM add-in version that matches the ArcGIS version installed on your computer.

\section{Installing Add-Ins in ArcMap}

The RLCM add-in can be downloaded from https://eros. usgs.gov/westafrica/tools-downloads. To install the add-in, follow these steps:

1. After downloading, double-click on the add-in file.

2. Click "Install Add-In" on the "Esri ArcGIS Add-In Installation Utility" screen. On a Windows 7 system, installed add-ins are stored in the default add-in folder:C: \Users $\backslash$ [User_Name]\Documents $\backslash$ ArcGIS $\backslash$ AddIns $\backslash$ Desktop10.x.

3. Click "Ok" at the installation succeeded prompt.

4. Open ArcMap. ArcMap automatically looks in the default add-in folder upon opening.

5. Choose "Customize," then the "Toolbars" tab, and check "RLCM Toolbar."

\section{Managing Add-Ins in ArcMap}

To manage add-ins in ArcMap, follow these steps:

1. Choose "Customize" and then "Add-In Manager."

2. In the "Add-In Manager" window a user can view and delete all the add-ins installed in their project.

\section{Setup and Description of the Rapid Land Cover Mapper}

This section describes the step-by-step procedures to setup and complete a mapping project using the RLCM add-in.

\section{The Rapid Land Cover Mapper Toolbar}

The "RLCM Toolbar" contains three buttons that each have a different function and one drop-down menu with two options (fig. 3):

- "Create Points and Time Periods" button-Define study area, point grid resolution, create points within study area, and manage mapping time periods.

- "RLCM Classes" button-Select classification system and associated land cover classes, and append selected land cover classes to the "RLCM Attributer."

- "RLCM Attributer" button —Attribute class to points.

- "Data Analysis" drop-down menu-Can choose from two options:

- "Create Rasters from Time Periods" option-Create raster from selected points for one or several time periods.

- "Create Change Rasters" option-Create change rasters from selected points or all points between two time periods.

\section{Create Points and Time Periods}

To create a map using the RLCM, the first step is to create a point grid feature class that will be used to complete the mapping. Before creating points, the data frame

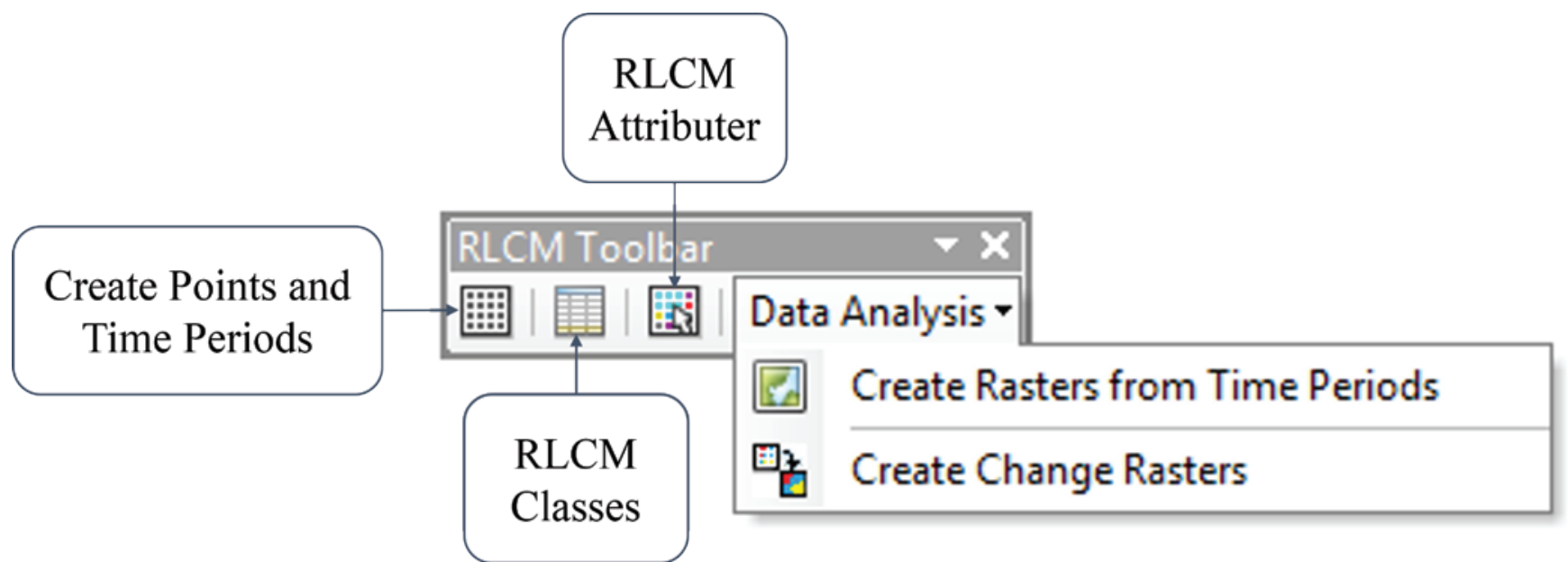

Figure 3. The "RLCM Toolbar" buttons and options. 
projection must be defined and the view extent scale must not exceed 1:800,000. Every ArcMap project contains a data frame, which displays a collection of layers in the table of contents. Right click on "Layers" at the top of the table of contents, click on "Properties," and select the "Coordinate System" tab. Check to make sure the current coordinate system box does not say "No Coordinate System."

Before creating points, the user must also determine a point resolution (the distance between each point) and mapping extent. The user must find the balance between the number of points desired for the mapping analysis, the level of precision of the output map (final resolution), the desired confidence level, and the level of effort that can be dedicated to the mapping exercise (see the "Define the RLCM Grid Resolution" section).

\section{Create Points}

1. On the "RLCM Toolbar," click on the "Create Points and Time Periods" button. The first time the RLCM add-in is used, the "RLCM Layer and Time Periods" dialog window is empty. If a RLCM layer is already present in the table of contents, the text boxes will be automatically populated (fig. 4).

2. Enter a study area name in the "Study area name" field. It will define the name of your RLCM layer. Entering the name of the study area is required and, once the RLCM layer is created, its name cannot be changed. $\stackrel{\Lambda}{\text { spacess }}$ The study area name must not contain underscores or

. The study area name is the base for using the RLCM add-in. A “_rlcm” suffix is automatically appended to the shapefile when the RLCM layer is created ([studyareaname] rlcm.shp). The suffix is used by the add-in to identify a valid RLCM layer.

3. In the "Output folder" field, define the RLCM workspace. The RLCM workspace is the folder in which all the required RLCM files will be stored. The user can choose to manually enter in the file path or click the folder icon to browse to the workspace location.

4. Optionally, a mask can be applied to the study area extent. The mask must be a polygon shapefile and must be added to the current ArcMap project to be displayed in the "Mask" drop-down list. The mask's coordinate system must be the same as the point layer. If a mask is selected, the points will be created only within the mask. If no mask is selected, the points will be created in the current ArcMap window extent.

5. Enter the point grid resolution of the RLCM layer in the "Point grid resolution" field. This value represents the distance in meters between each point to be created.

6. Click on the "Create Points" button. A new RLCM layer will be created, stored in the output folder, and automatically added to the table of contents in ArcMap.

! The user does not have to create all the points within the study area at once. Once the RLCM layer is created, new points can easily be added from the "RLCM Attributer" window (see the "Overview of the RLCM Attributer" section).

\section{Define Time Period(s)}

Another requirement of the RLCM add-in is to specify time period(s) relative to the imagery used for mapping:

1. In the "RLCM Layer and Time Periods" window, click on the "Add Time Period" button in the lower left corner. A blank field and a "Save Time Period" button will appear (fig. 5).

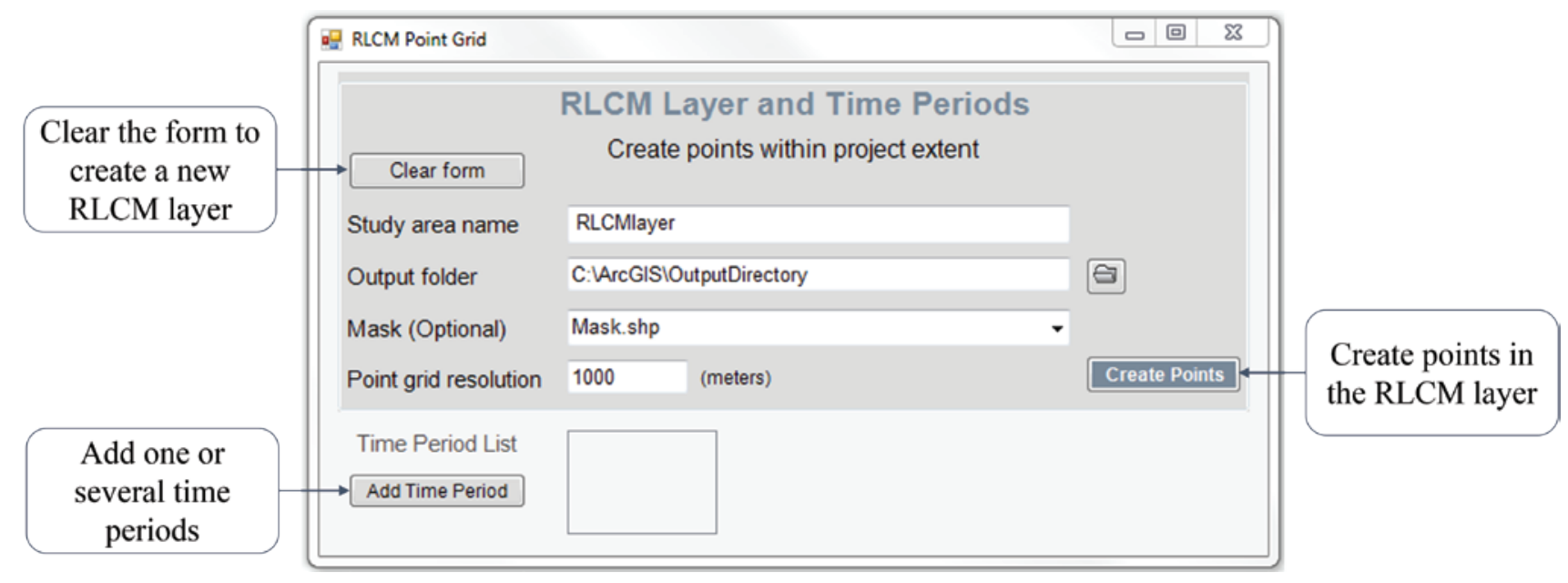

Figure 4. The "RLCM Layer and Time Periods" window. 
2. Enter a year in the field to define a time period. Time periods can range from a single year to decades, there is no limit to the number of time periods (fig. 5).

! The "Year" represents a time period that characterizes the date range of the imagery used for mapping. Good quality images may not be available for the same year over the whole extent of the study area, and the user may need to use images from multiple years to complete the coverage. For instance, a map of 2010 land cover may be based on imagery from 2008 to 2012 .

3. Click "Save Time Period." The time period is added to the "Time Period List" box.

4. Close the "RLCM Layer and Time Periods" window.

\section{Rapid Land Cover Mapper Classes}

Once the RLCM layer is created, click on the "RLCM Classes" button from the "RLCM Toolbar" to open the "RLCM Classification System" window (fig. 6).

\section{Visualize and Choose a Classification System}

The final step before starting mapping is to select a land cover classification system and associated classes from the "RLCM Classification System" window (fig. 6). In this version, the RLCM add-in is delivered with seven embedded classification systems described in appendix 1. Follow these steps to select a classification system:

1. Select the RLCM layer from the drop-down list. If there is only one RLCM layer in the table of contents, it will be selected by default.

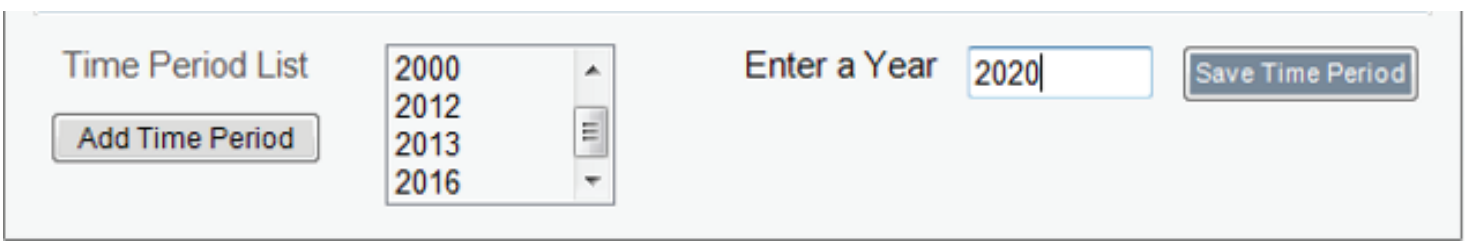

Figure 5. Add a time period from the "RLCM Layer and Time Periods" window.

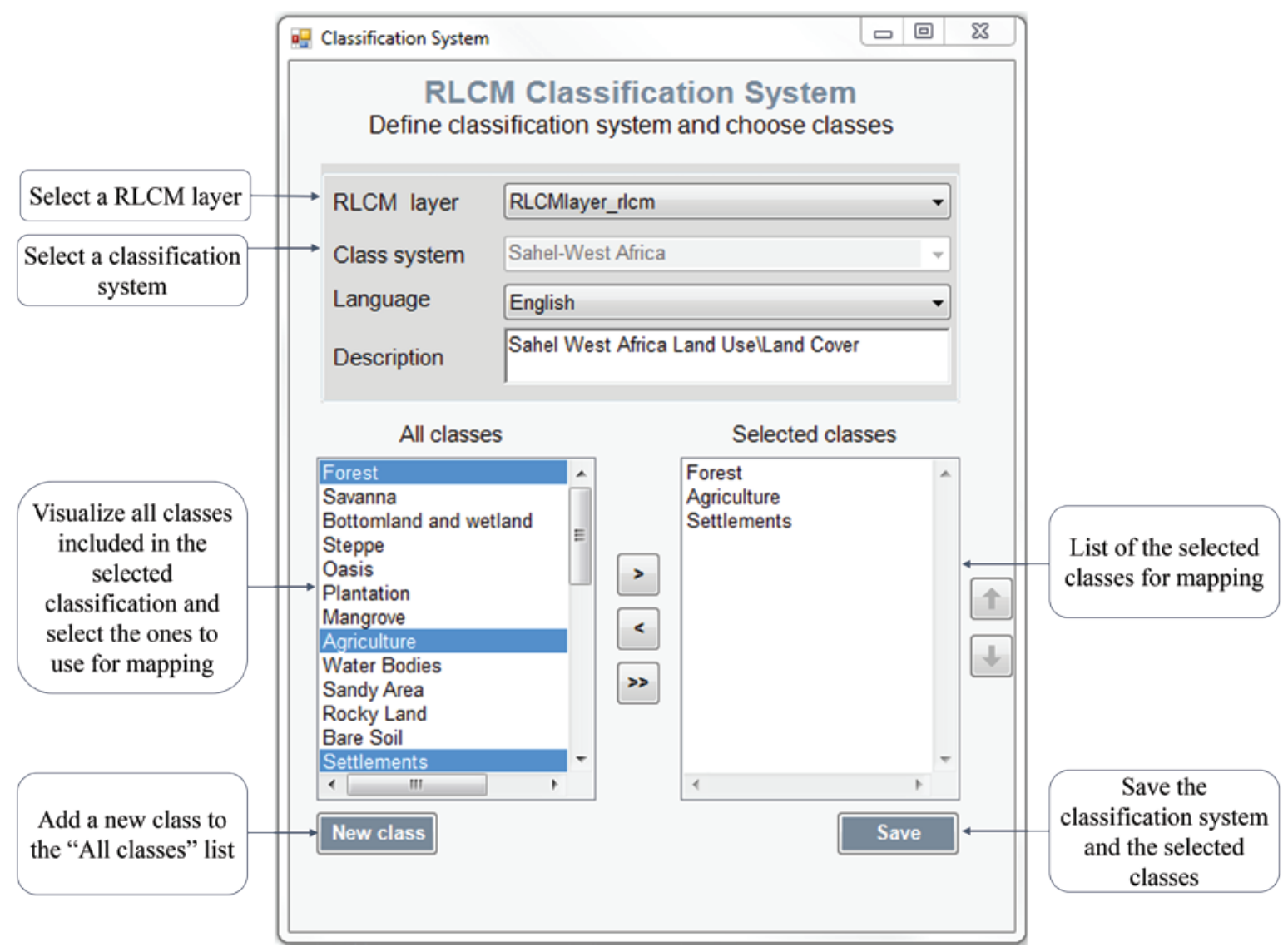

Figure 6. The "RLCM Classification System" window. 
2. Choose a classification system from the "Class System" drop-down list. A description of the selected classification system is shown in the "Description" field. When a classification system is selected, the list of associated classes is displayed in the "All classes" list box. The user can change and explore different classification systems.

3. Choose the "Language" of the classes. By default, the language is English, but some classification systems are bilingual (English and French) (see appendix 1).

4. To select classes from the "All classes" list box, highlight them and click on the right arrow button $(>)$. To select multiple classes at once, press and hold the control key while clicking on classes. These selected classes will be added to the "Selected classes" list box and will be displayed in the "RLCM Attributer" window to be used for mapping. This functionality allows the user to minimize the number of classes to choose from while mapping. If some classes are not present in the study area, there is no reason to display them in the "RLCM Attributer" window, and removing them reduces the potential for misclassification. The classes can be reordered using the grey up and down arrows. The order will be reflected in the "RLCM Attributer" window. The "Selected classes" can be appended to at any time during the classification processes.

$\widehat{\Lambda}$ Once a class is selected, the classification system is locked and cannot be changed.

5. To add a new class to the "All classes" list, click on the "New Class" button. A form will appear to append an additional class (fig. 7). See the "Create a Custom Classification System" section for the description and requirements to define a new class.

6. Click on "Save" to save the selected classification system and classes.

\section{Create a Custom Classification System}

If none of the provided classification systems meets the user's requirements, the RLCM add-in allows the user to build a custom classification.

In the "RLCM Classification System" window, choose "Custom" from the "Class System" drop-down list and manually enter the classes using the "New Class" button following the class attributes requirements, as described below. Each class must be defined by three attributes (see appendix 1):

- Rlcm_class_no-Unique integer for each class within a class system that is automatically generated when the user creates a new class; for example, 1, 2, 3...

- Code-Unique code for each class (one to three characters); for example, "F" for Forest, "Sa" for Savanna, and "Urb" for Urban.

- Class name-Unique short name describing the class; for example, Forest, Savanna, or Urban

A Any modification or addition made to the classification system is specific to the project and the associated RLCM layer. A custom classification system will not be saved within the RLCM add-in.

\section{Mapping with the Rapid Land Cover Mapper Add-In}

Once the RLCM layer is created, and the time periods and the classification system are chosen, the user can start classifying the points.

\section{The Rapid Land Cover Mapper Layer}

\section{Layer Projection}

Based on the study area location, the RLCM layer projection is automatically generated when the layer is created. The RLCM's custom projected coordinate system divides the world into nine zones and is based on the Lambert Azimuthal Equal Area projection (meters-based projection) (see appendix 2). The coordinate system of the RLCM layer must not be changed. The RLCM add-in will not function properly if the projection is changed.

The RLCM layer is the only dataset required to use this predefined custom projection coordinate system. Often, the ArcMap project's data frame projection is different than the RLCM layer projection; when this happens, the RLCM points may appear shifted. Refer to the "Frequently Asked Questions" section to adjust the data frame coordinate system if needed.

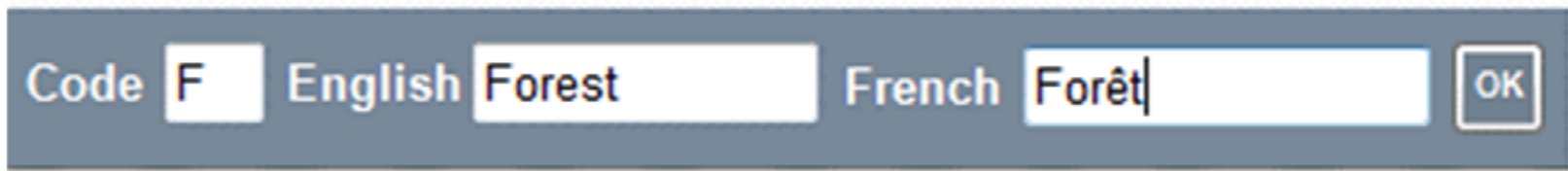

Figure 7. Add a new class form. 


\section{Attribute Table}

Once the RLCM layer is created, four attribute fields are automatically generated in the attribute table (fig. 8):

- "xyp" field-The X and Y coordinate of each point, calculated when the point is created.

- "LC_Nm[year1]" field-The unique class name attributed to each point, for the time period [year1].

- "LC_Cd[year1]" field-The unique code associated with the class attributed to each point, for the time period [year1].

- "LC_Id[year1]" field—The unique integer associated with the class attributed to each point, for the time period [year1].

\section{Overview of the Rapid Land Cover Mapper} Attributer

To begin classifying the points, open the "RLCM Attributer" window (fig. 9) by clicking on the "RLCM Attributer" button in the "RLCM Toolbar." The "RLCM layer" dropdown list, the "Year" drop-down list, and the selected classes" field are automatically populated.

\section{Changing and Saving the Points Symbology}

The first time the "RLCM Attributer" window (fig. 9) is opened, a default symbology (symbols and colors) is assigned to each selected class of the RLCM layer and saved in an ArcGIS layer file (*.lyr) named "[studyareaname]_rlcm.lyr" in the user's RLCM workspace. The user can change the color and symbol of each class (fig. 10).

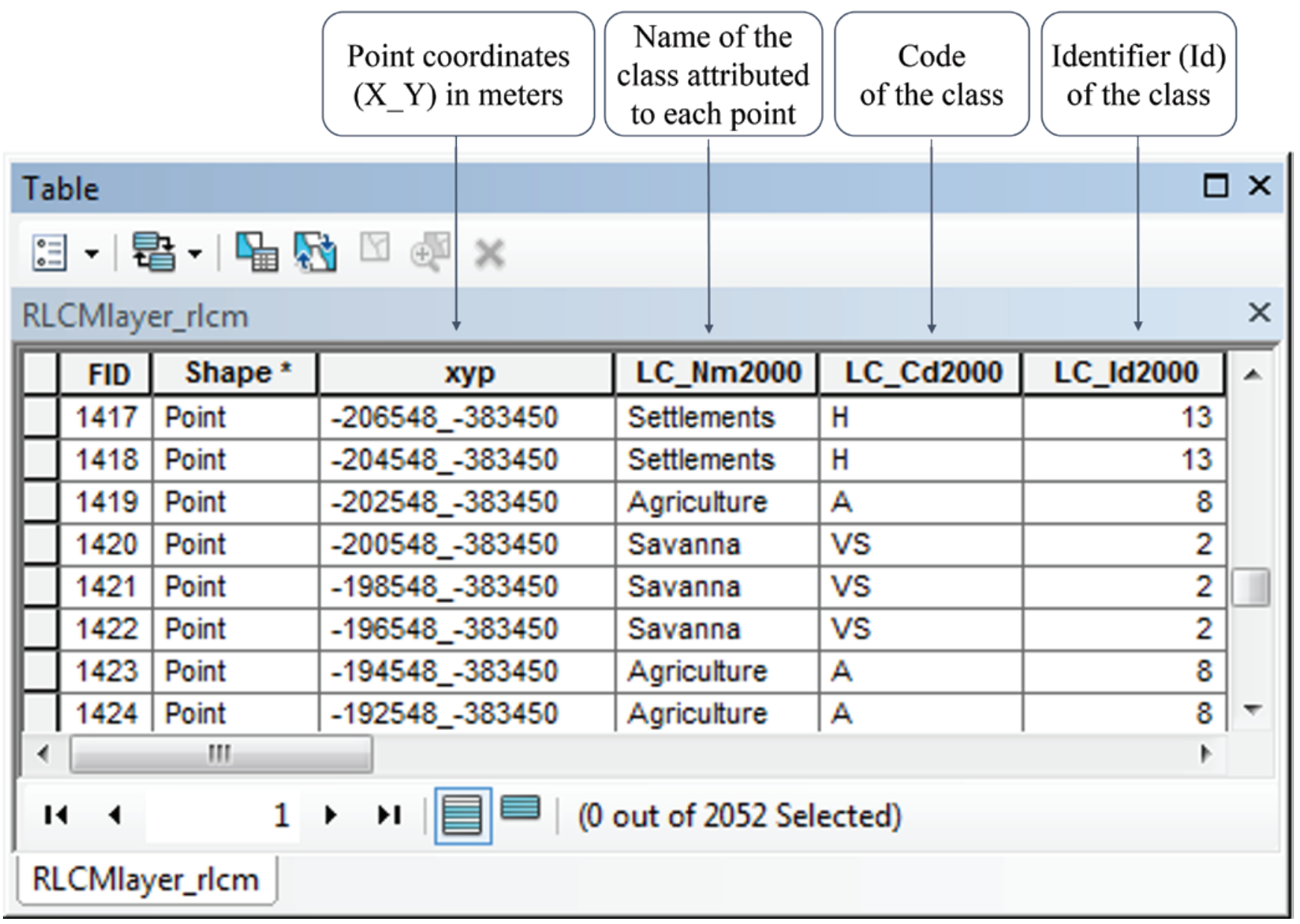

Figure 8. Example of attributed points in the RLCM layer attribute table for one time period (2000). 


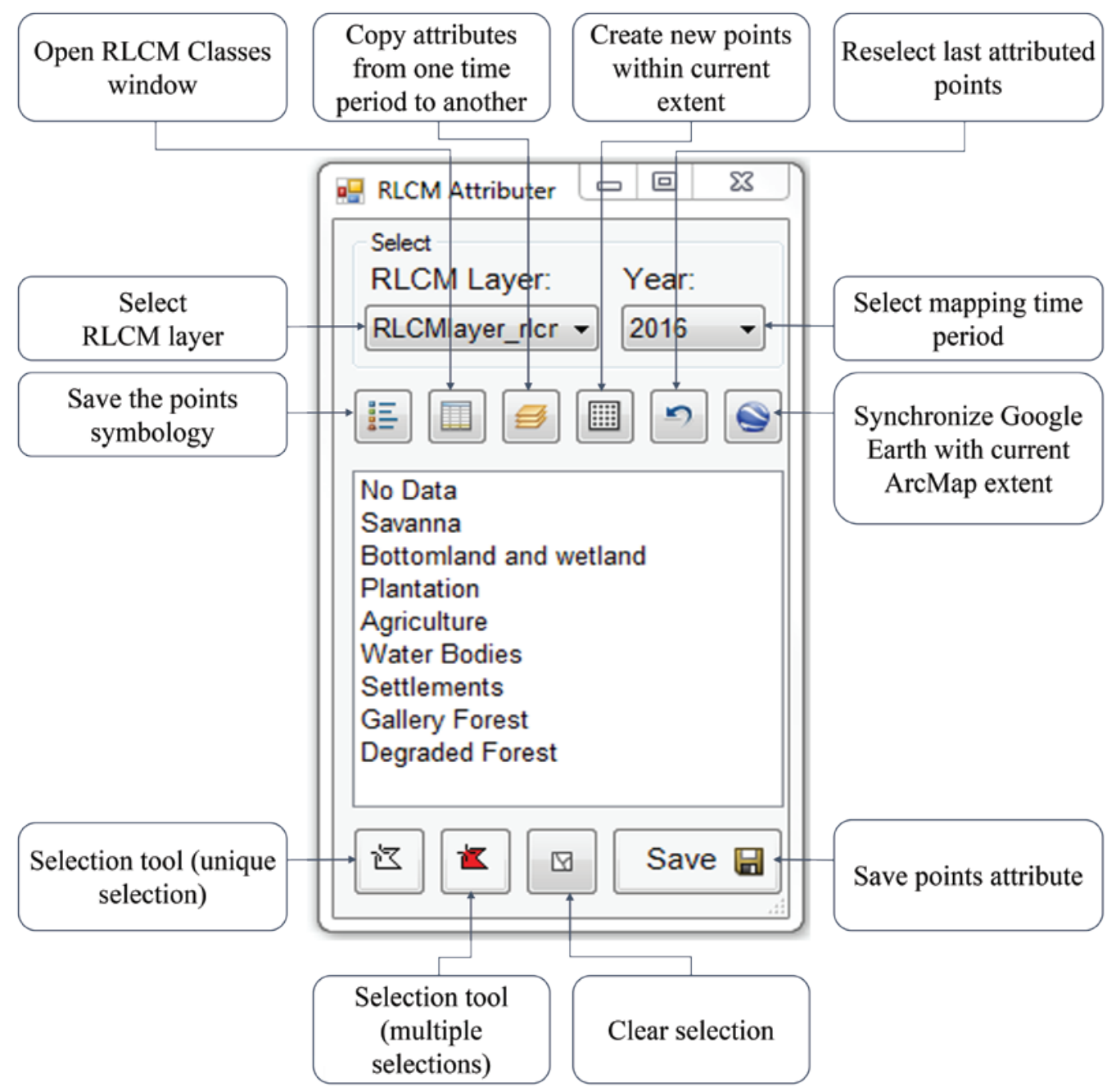

Figure 9. The "RLCM Attributer" window.

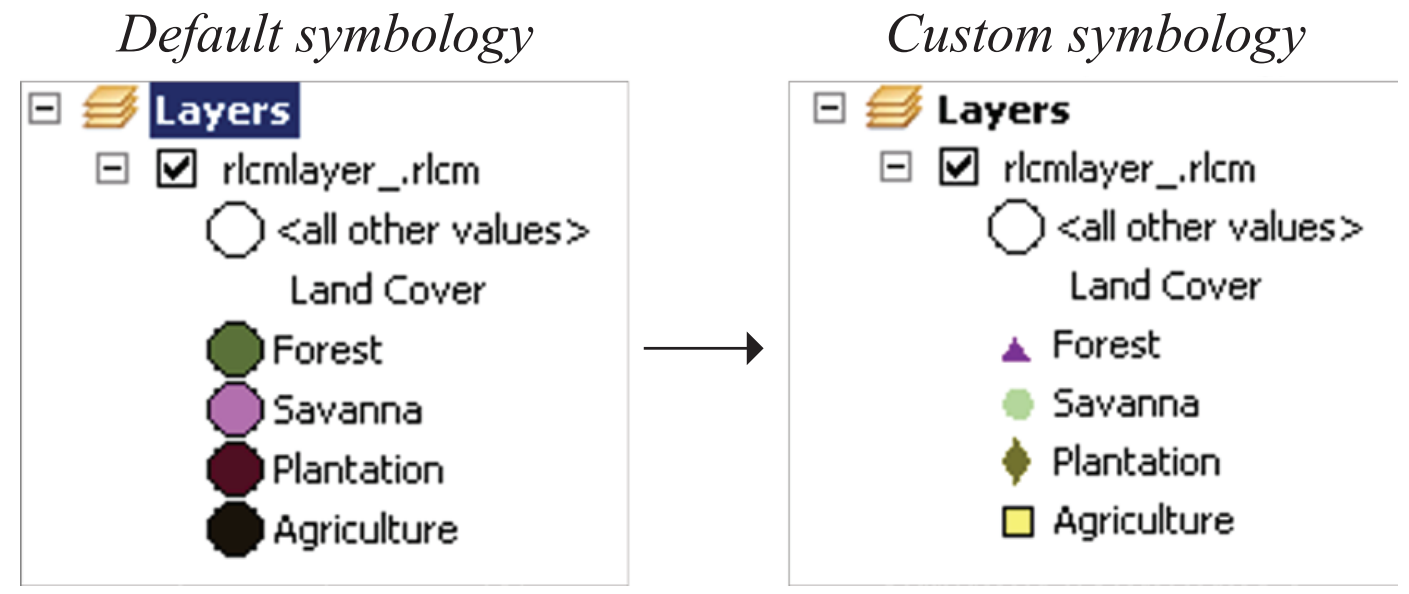

Figure 10. Examples of default and custom symbologies for several land cover classes. 
To save a user's custom symbology, click on the "Save

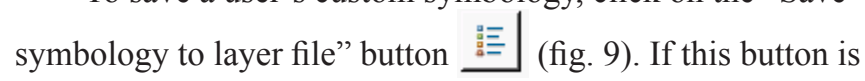
not clicked and the user closes the project, the point symbols and colors will not be saved. The colors and symbols will default back to a random color ramp.

\section{Selecting and Attributing Points}

Using one of the selection tools from the "RLCM Attributer" window, click and drag the cursor to select points. Choose the associated land cover class in the "RLCM Attributer" window and click "Save" (fig. 11). Repeat until all the points are attributed.

\section{Changing Time Period}

A key function of the RLCM add-in is to provide a method for mapping multiple time periods at a single location, using a cascading period classification. In the setup, the user creates an initial time period, but at any time additional time periods can be created (see the "Define Time Period(s)" section). Cascading is a method of completing the classification of a time period for a given area, then pushing that classification information forward or back in time.

From the "RLCM Attributer" window (fig. 9), click on the "Copy Time Period" button $\cong$ to open the "Copy Time Period" window (fig. 12). Follow these steps to copy attributes from one time period to another:

1. Select the points to copy using one of the selection tools.

2. Choose the time period 1 (mapped) and time period 2 (not mapped yet).

3. Click on "Copy." The progress bar shows when the copy is completed.

After copying the attributed points into another time period, they are displayed over images that correspond to the "new" time period (fig. 13). The interpretation process is repeated, and in this case the previously attributed points are reviewed to determine if they should remain unchanged or be edited to reflect a change in the image. Generally, the image analyst begins with the most recent time period, then works back in time to earlier time periods.

I When mapping multiple time periods, it is recommended to start mapping the most recent time period first (for instance, 2010 versus 1976). High-resolution imagery or online highresolution map services are freely available to verify the accuracy of the classified points.

\section{From the Rapid Land Cover Mapper Layer to Output Maps}

Once the classification is completed in the study area, the user can create raster datasets for analysis. To complete an analysis using the RLCM add-in, click on the "Data Analysis" menu in the "RLCM Toolbar" (figs. 3 and 14).

\section{From the Rapid Land Cover Mapper Layer to a Raster Map}

The "Create Rasters from Time Periods" option (figs. 3 and 14) allows users to create one or several rasters from the entire RLCM layer or for a selection of points.

1. Click on the "Data Analysis" drop-down menu on the "RLCM Toolbar," and select "Create Rasters from Time Periods."

2. Select one or multiple time periods in the "Time period" list box (fig. 15). To select multiple time periods at once, press and hold the control key while clicking on time periods (years).

3. Click on the "Create Rasters" button.

4. One or multiple rasters named "[studyareaname]_[year]. tif" will be automatically added to the table of contents (fig. 16).
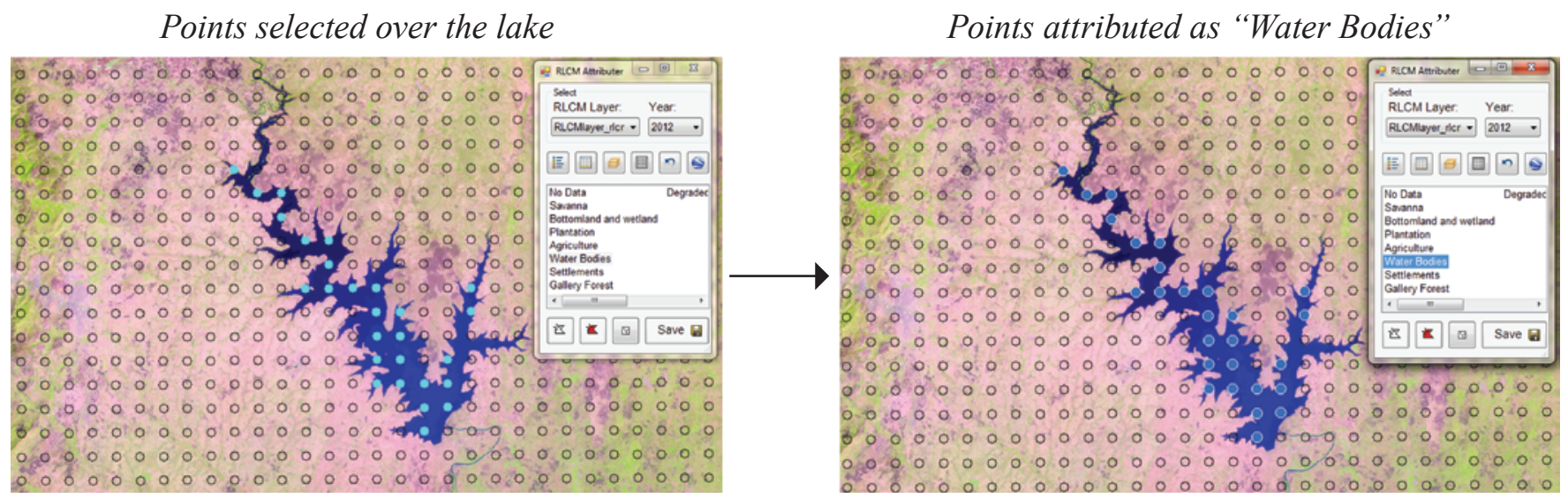

Figure 11. Selecting and attributing points with common land cover class. 


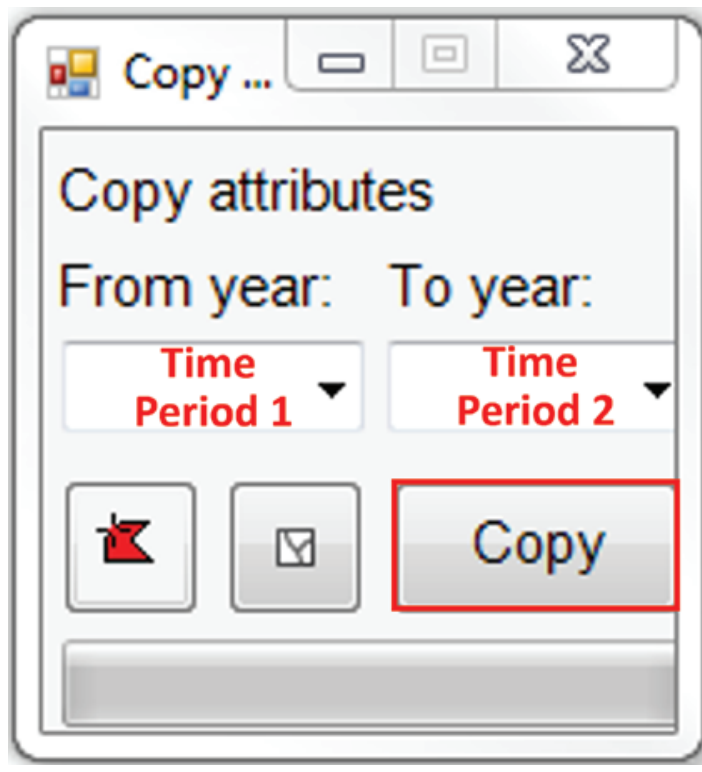

Figure 12. Copy attributes from one time period to another.
The attribute table of the generated raster contains the following fields (fig. 17):

- "LC_Nm[Year]" field-The descriptive class name.

- "Area(sqkm)" field-The area covered by each class, in square kilometers.

- "LC_Id[Year]" field-The identifier associated with each class.

- "LC_Cd[Year]" field_-The code associated with each class.

For more information about the code and the identification of each class, see the "Create a Custom Classification System" section.

\section{From the Rapid Land Cover Mapper Layer to a Change} Map

The purpose of time-series mapping is to identify changes between time periods. To create change maps, click on the

\section{Attribute all the points \\ for time period 1 (here, 2000)

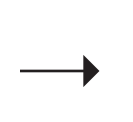 \\ Select all the attributed points \\ and copy attributes from time period 1 (here, 2000) to \\ time period 2 (here, 1976)}

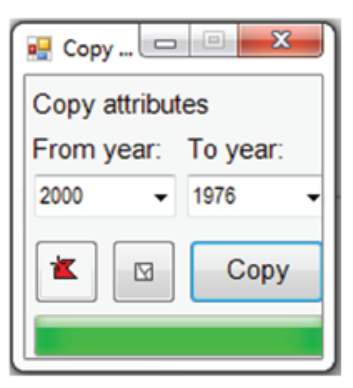

Attribute all the points with the corrected classes for time period 2
Load time period 2 imagery (here, Landsat MSS 1976), and overlay time period 2 attributed points (here, 1976) by selecting the associated "Year" in the Attributor dropdown box

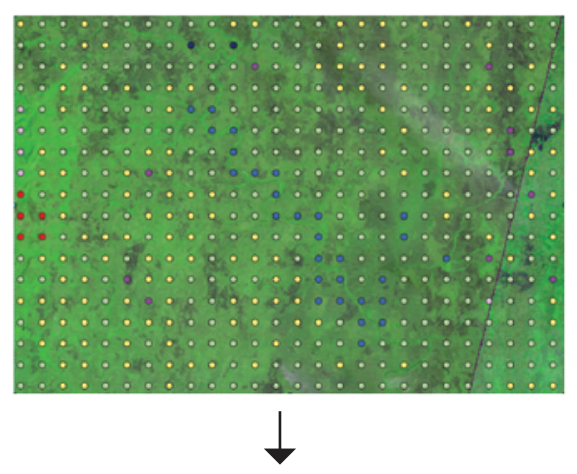

Select points that have changed and re-attribute them with the correct class
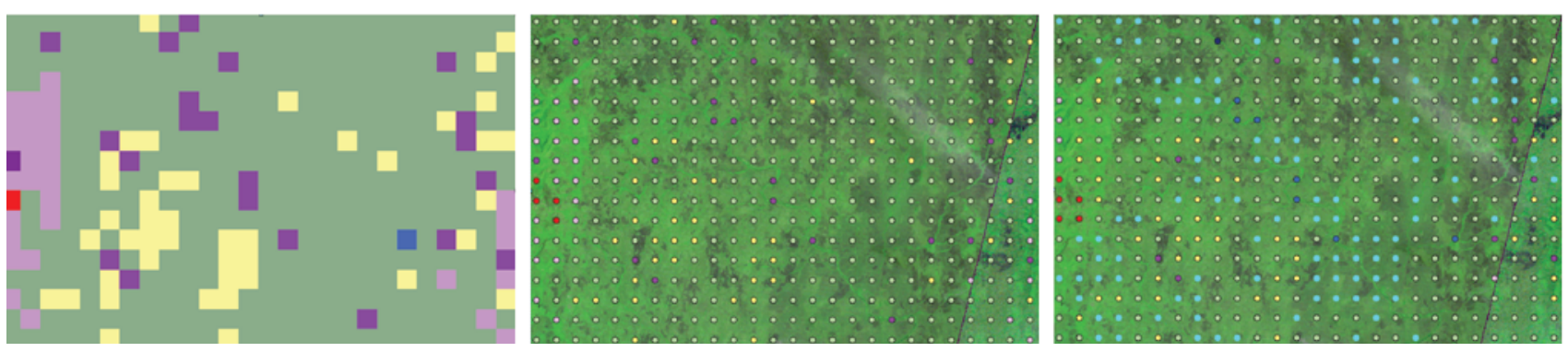

Figure 13. Process for quick multiple-time period mapping using the Rapid Land Cover Mapper. 


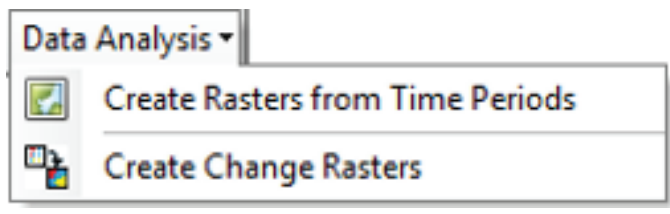

Figure 14. The "Data Analysis" drop-down menu.

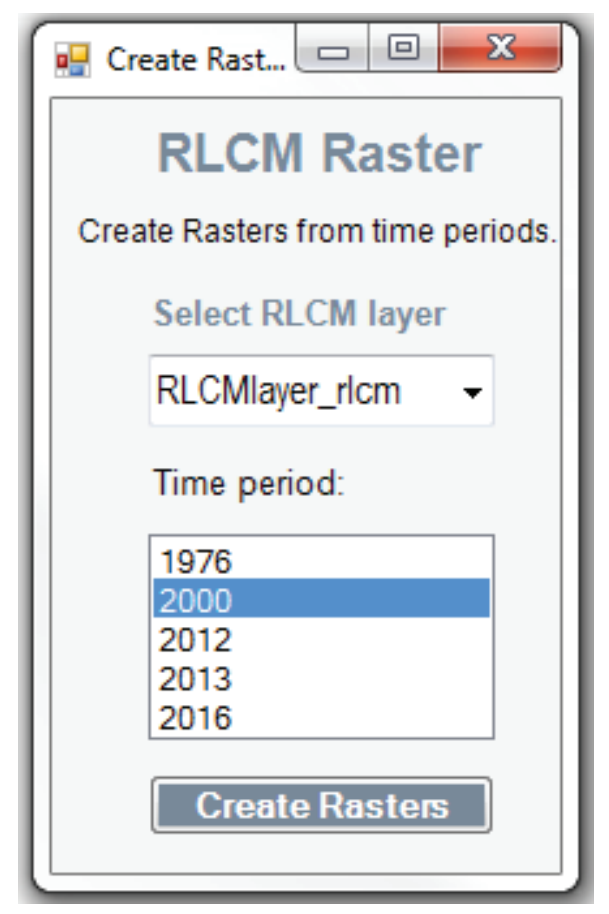

Figure 15. The "Create Raster from Time Periods" window.
"Data Analysis" drop-down menu and select "Create Change Rasters" (figs. 3 and 14).

1. In the "RLCM Change Map" window (fig. 18), select the time periods between which to analyze the changes ("from" and "to" time periods).

2. Select one or multiple classes in the two list boxes. To select multiple classes at once, press and hold the control key while clicking on classes. To select all classes at once, click on the "Select all classes" check box. To select all classes but one, choose the class to exclude from the selection using the "Select all classes BUT" drop down list.

3. Click on the "Create change raster" button.

The output raster will contain only the pixels that represent the change from the selected classes and time periods (fig. 19).

\section{Behind the Scenes-Rapid Land Cover Mapper Required Files}

\section{XML File}

When a RLCM layer is created, an .xml file ([studyareaname]_rlcm.xml) is also created in the output folder.

A The .xml file is the core of the RLCM tool and should never be deleted. The .xml file stores all the inputs required for the tool to work properly. A detailed example of an .xml file is provided in appendix 3 . The .xml file includes the following information:

1. Language-Records the default class language selected by the user in the "RLCM Classification System" window (see the "Rapid Land Cover Mapper Classes" section).
From the RLCM point layer

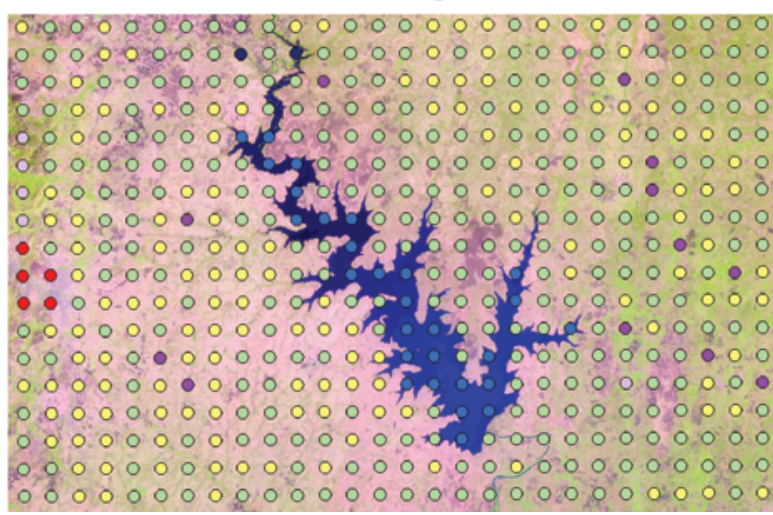

To the output raster map (2,000 meters resolution)

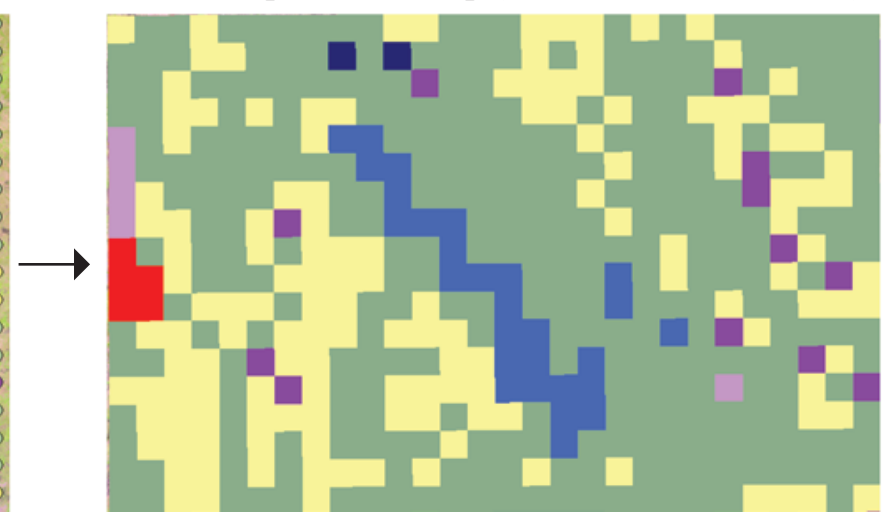

Figure 16. Example of the conversion of a Rapid Land Cover Mapper point layer to a raster map. 


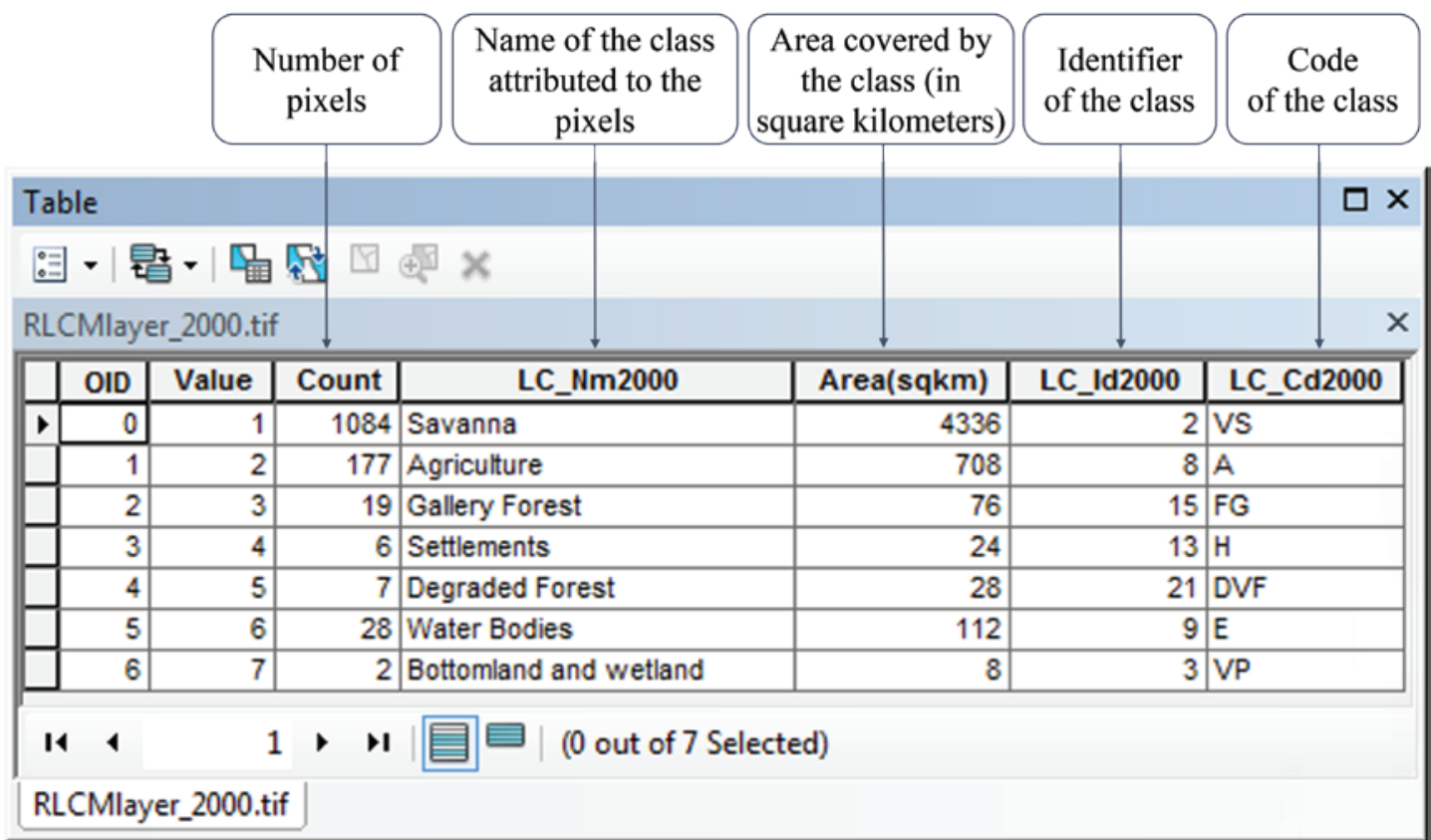

Figure 17. Example of the attribute table of a Rapid Land Cover Mapper raster for the year 2000.

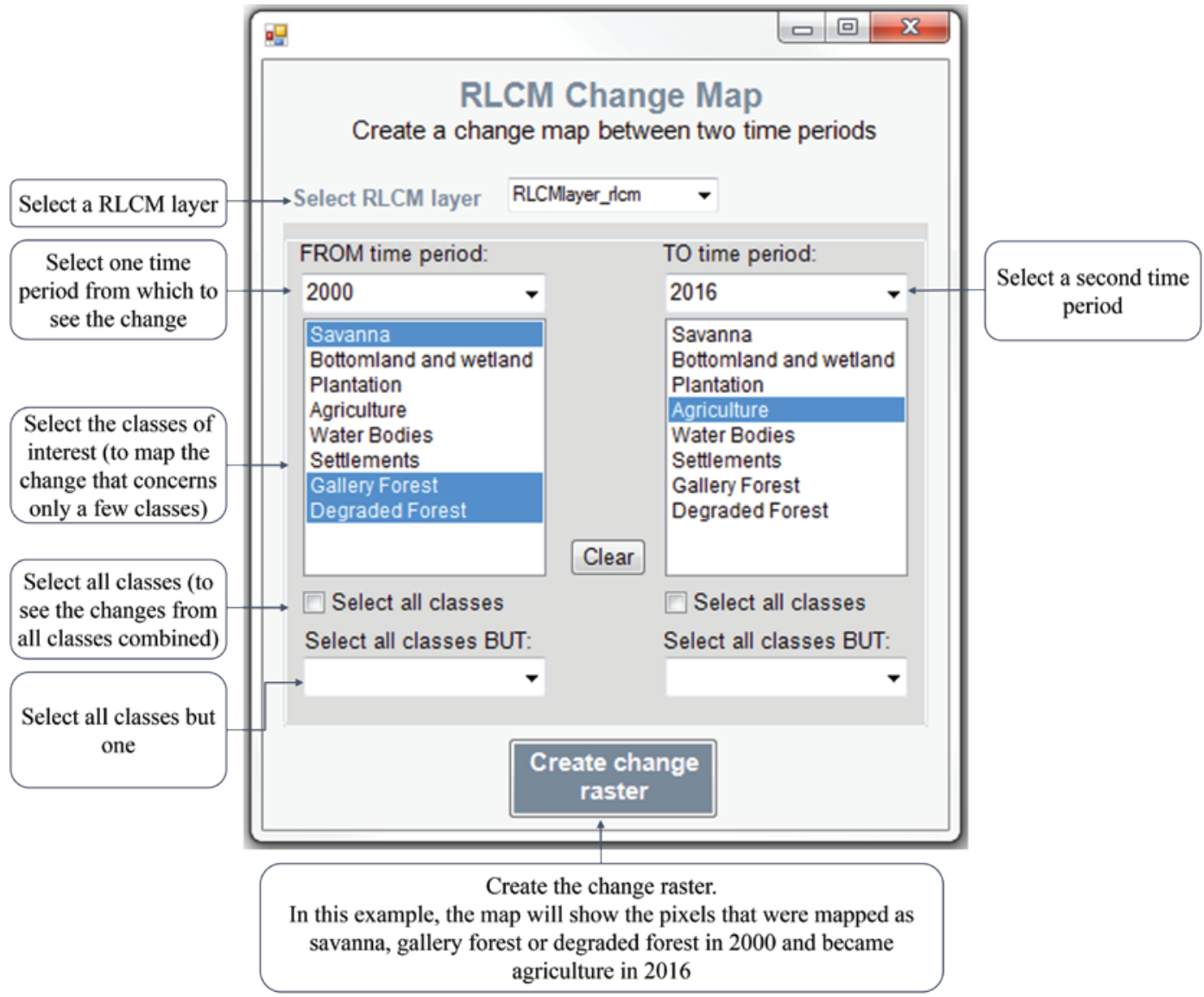

Figure 18. The "RLCM Change Map" window. 

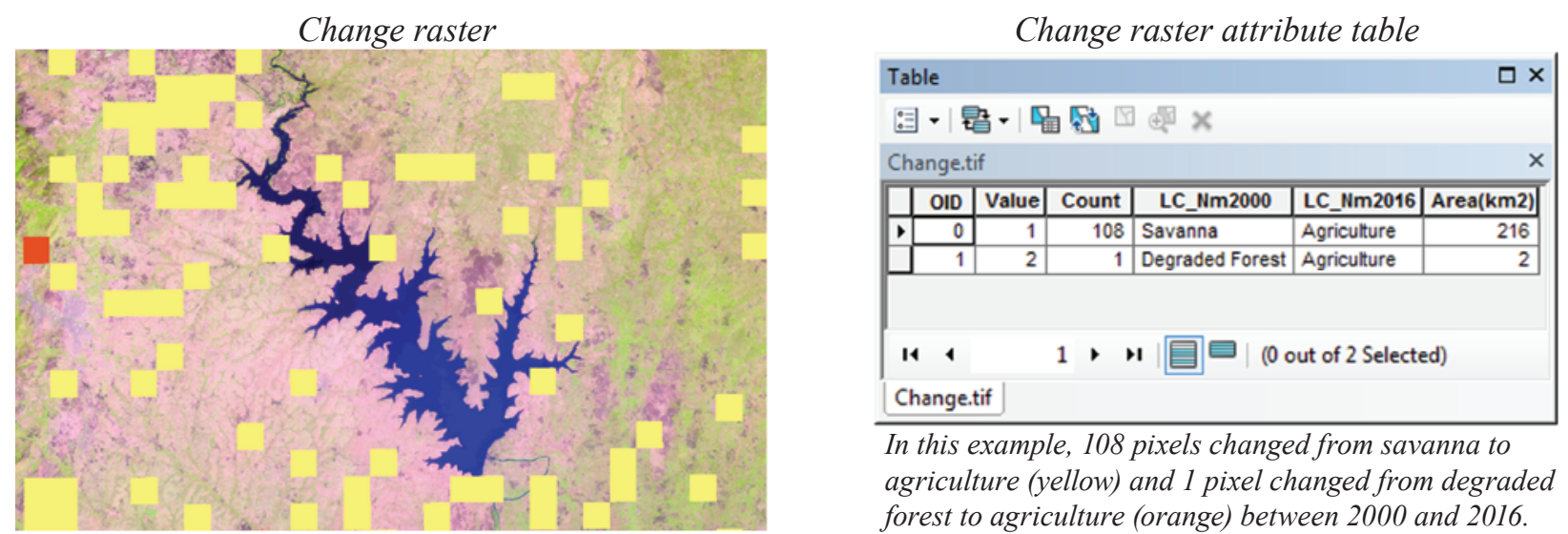

Figure 19. Example of a change raster and associated attribute table.

2. RLCM Dots-Records the inputs from the "RLCM Layers and Time Periods" window (see the "Create Points and Time Periods" section).

3. TimePeriods - Records each time period added to the project (see the "Define Time Period(s)" section).

4. ToolConfig-These tags configure display options for the RLCM layer and can be changed by the user.

- BuildShapeIndex-If "True," an index is automatically built in the attribute table. It speeds up the selection and attributing of the points. This option works well for large datasets (more than 30,000 points).

- DotCreationScale-Defines the maximum scale to create points. By default, the scale is set to $1: 800,000$.

- Class_System-Records the classification system and the classes selected by the user in the "RLCM Classification System" window (see the "Rapid Land Cover Mapper Classes" section).

The user must not edit any tags directly in the .xml file, except the "ToolConfig" and "Class_System" tags.

\section{Layer File}

When a RLCM layer is created a .lyr file ([studyareaname]_rlcm.lyr) is added in the output folder. This .lyr file records the symbology of the RLCM layer every time a point is attributed or when the user clicks on the "Save symbology" button from the "RLCM Attributer" window (fig. 9) (see the "Changing and Saving the Points Symbology" section).

\section{Selection Memory Text File}

The feature identifier values of the last attributed points are saved in the "SelectionMemory.txt" file in the output folder. It allows the user to reselect these points using the "Reselect last attributed points" button in the "RLCM Attributer" window (fig. 9) (see the "Overview of the RLCM Attributer" section). The text file is updated after point attributes are saved in the "RLCM Attributer" window.

\section{Rapid Land Cover Mapper Tips}

Some tips are described below to help use the tool more efficiently and facilitate the mapping process.

\section{Check Imagery Availability}

The RLCM add-in works with all image formats supported by Esri's ArcMap. The availability of the imagery can help the user define time periods for mapping. Good quality images might not be available for the same year over the whole extent of the study area, and the user might need to use images from multiple years to complete the coverage. For instance, a map of 2010 land cover may be based on imagery from 2008 to 2012, and a map of 2000 land cover on imagery from 1999 to 2004 .

\section{Define the Rapid Land Cover Mapper Grid Resolution}

As stated before, the user must find the balance between the number of points desired for the mapping analysis, the level of precision of the output map (final resolution), the 
desired confidence level, and the level of effort that can be dedicated to the mapping exercise. The RLCM grid resolution should be based on the extent of the study area and the imagery resolution (table 1).

\section{Keep Track of the Mapping Progress}

If you are working with more than one time period, all the points for both time periods will be attributed after copying the point's attributes from time period 1 to time period 2 (see the "Changing Time Period" section). When starting mapping for time period 2, it can be difficult to keep track of the mapping progress (that is, correcting all the points that changed between time period 1 and time period 2). Drawing uniform colored graphic boxes will help keep track of mapping progress (fig. 20). To add a box, make sure the "Drawing" toolbar is visible, and click on the "Rectangle" drawing tool. Change the fill color to no color and adjust the line color.

\section{Synchronize View Extent with Google Earth}

When mapping land cover for a recent time period, Google Earth is a valuable resource to quickly access highresolution imagery. Before using the Google Earth button on the "RLCM Attributer" window, make sure Google Earth is open and running. Click on the "Google Earth" button in the "RLCM Attributer" window and Google Earth will zoom to the same location as the ArcMap's view extent.

\section{Frequently Asked Questions}

\section{How do I Add or Delete Points?}

- To add new points, (1) click on the "Create Points and Time Periods" button in the "RLCM Toolbar" (fig. 3) (see the "Rapid Land Cover Mapper Toolbar" section) and click on "Create Points" (fig. 4), or (2) click on the "Create Points" button in the "RLCM Attributer" window (fig. 9) (see the "Overview of the Rapid Land Cover Mapper Attributer" section).

- To delete existing points, toggle the RLCM layer into editing mode, select the points to delete, delete them (see the Deleting Records in a Table), save the changes, and stop the editing mode.

\section{How do I Add or Remove a Time Period?}

- To add a new time period, click on the "Create Points and Time Periods" button in the "RLCM Toolbar" (fig. 3), click on "Add Time Period" (fig. 4), and enter a new time period (see the "Define Time Period(s)" section).

- To remove an existing time period, follow these steps:

1. Delete the fields associated with the time period from the RLCM layer attribute table (see the "Attribute Table" section): In the ArcToolbox

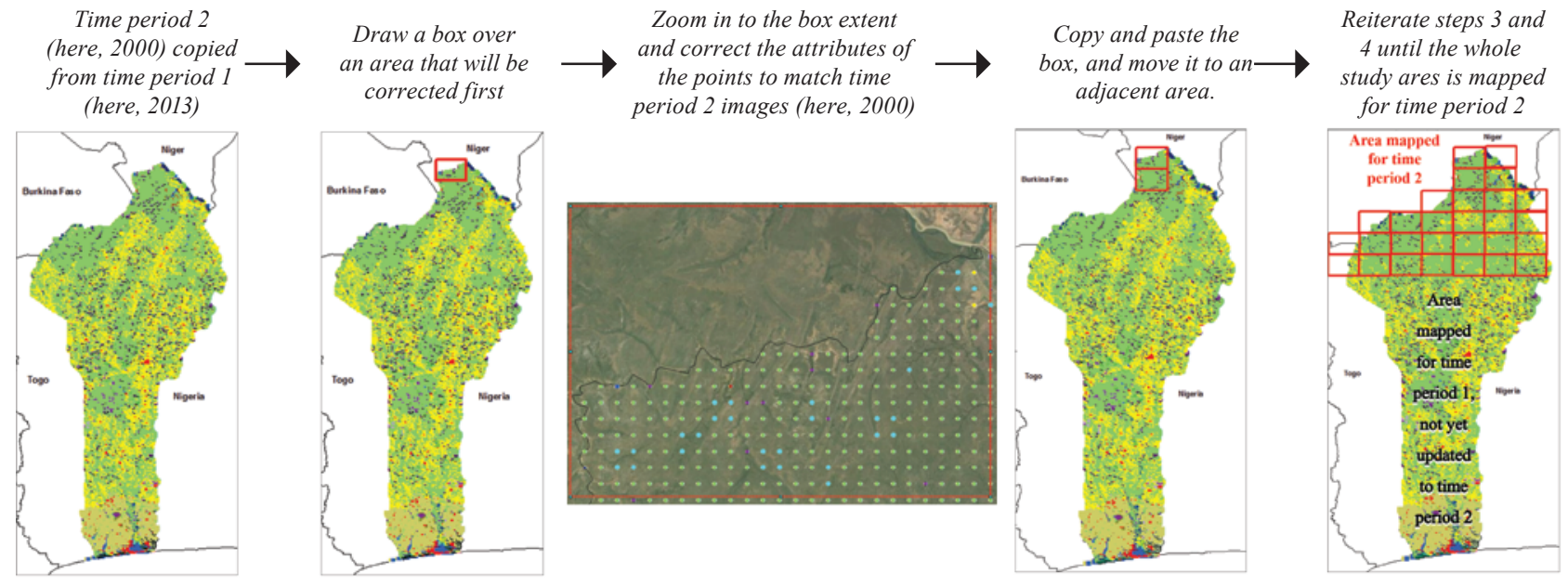

Figure 20. Tip to keep track of the multiple time period's mapping progress using the example of land cover mapping in Benin. 
Table 1. Suggested imagery source and number of points in the point grid based on points resolution and study area size.

[Blue shading indicates high resolution images ( 1 to 5 meter resolution); gray shading indicates number of points too high or too low; green shading indicates Advanced Spaceborne Thermal Emission and Reflection Radiometer or SPOT images (10 to 20 meter resolution); orange shading indicates Landsat images (30 to 60 meter resolution)]

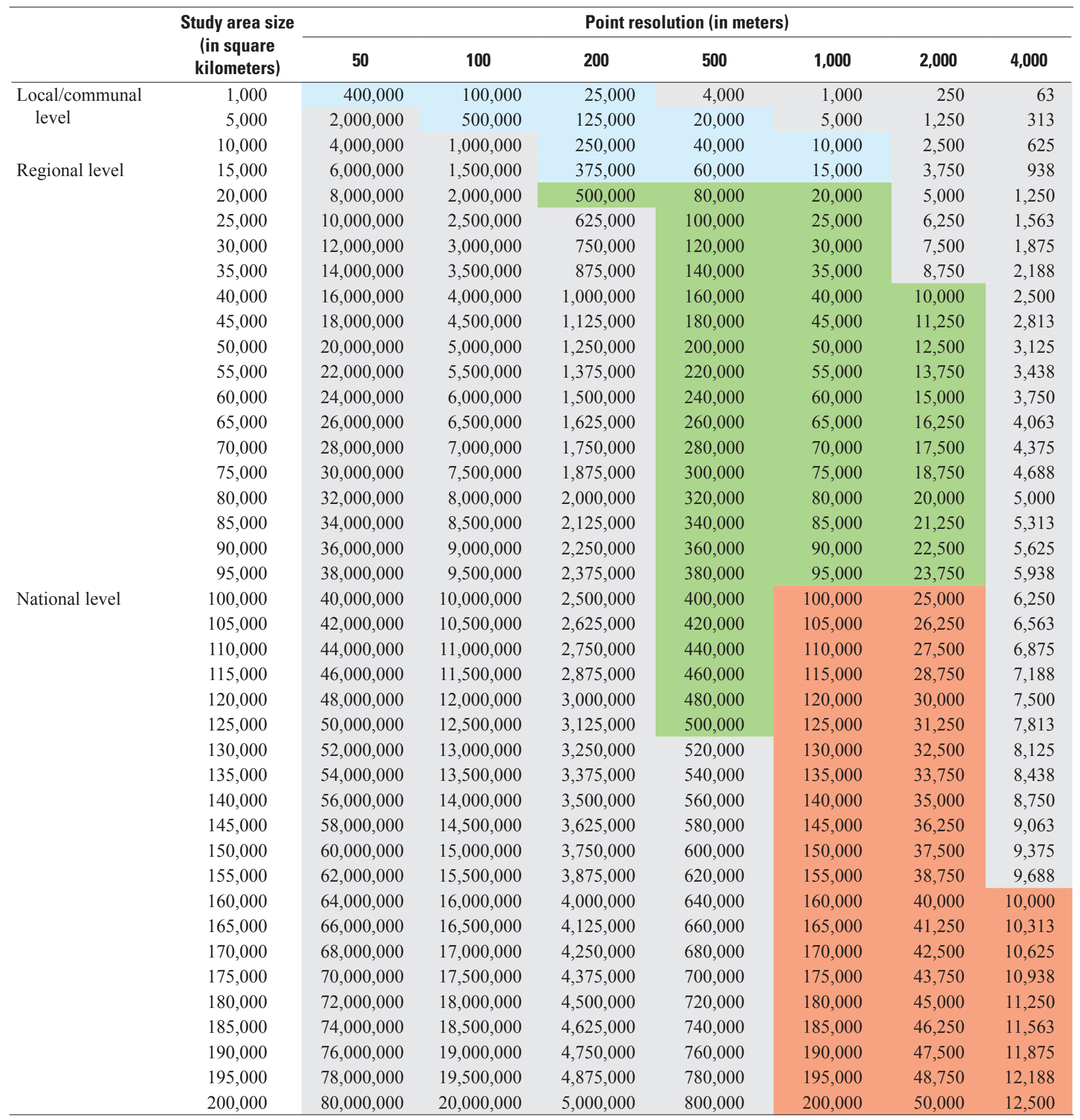


$>$ Data Management Tools $>$ Fields $>$ Delete Field, select the three fields associated with the time period (LC_Nm[year], LC_Cd[year], and LC Id[year]), and click "OK."

2. Delete the time period record from the RLCM layer .xml file: Navigate to the output folder and open the "[studyareaname]_rlcm.xml" file with Notepad.

A Before editing the .xml file, make a copy of it.

3. Select the time period to delete, including the associated tags $<$ TimePeriod $>$ and $</$ TimePeriod $>$. Delete these lines (fig. 21) and save the .xml file (for more details on the .xml file, see the "XML File" section and appendix 3).

\section{How do I Add or Remove a Class From the "RLCM Attributer" Window?}

1. To add a class from the "RLCM Attributer" window (fig. 9), click on the "RLCM classes" button, select the class to add from the "All classes" list box, click on the right selection arrow $(>)$ to add the class to the "Selected classes" list box, and click "Save" (fig. 6) (see the "Visualize and Choose a Classification System" section).

2. To remove a class from the "RLCM Attributer" window (fig. 9), click on the "RLCM classes" button, select the class to delete from the "Selected classes" list box, click on the left selection arrow $(<)$ to remove the class from the "Selected classes" list box, and click "Save" (fig. 6) (see the "Visualize and Choose a Classification System" section).

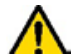
Before deleting a class from the "RLCM Attributer" window, make sure no points were attributed to this class.

\section{How do I Add or Delete a Class to a Classification System?}

1. To add a new class to an existing classification system (into the "All classes" list box), click on the "New Class" button (fig. 6). Fill in the form to create a new class (fig. 7) (see the "Visualize and Choose a Classification System" section).

2. It is not possible to delete a class from a classification system.

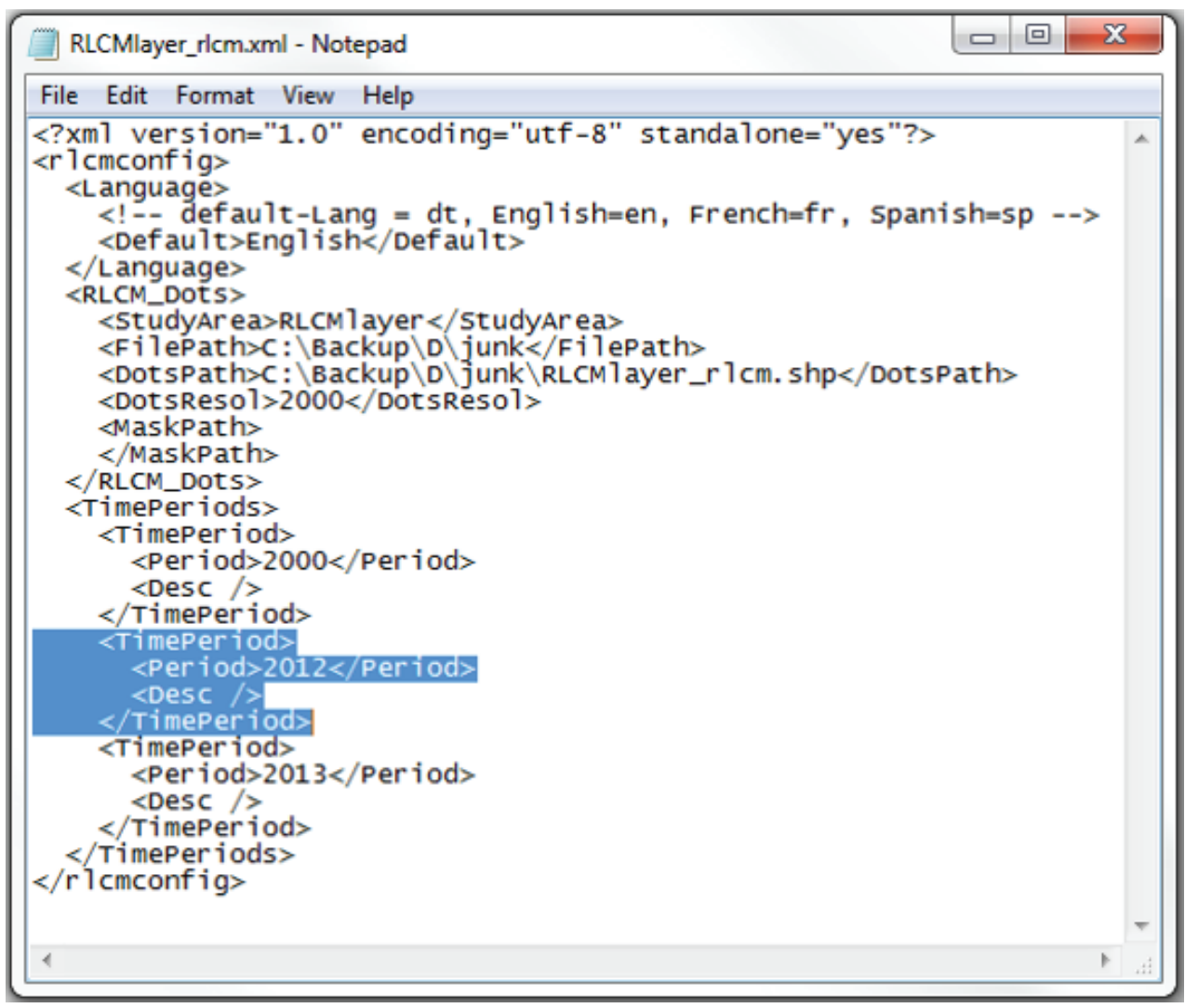

Figure 21. Time period record in the .xml file of the Rapid Land Cover Mapper layer. 


\section{Why do my Points Appear Shifted?}

The points of the RLCM layer can appear shifted in ArcMap if the data frame projection is different from the custom RLCM layer projection. To fix the shifted points, the user needs to change the data frame projection to the same projection as the RLCM layer (figs. 22 and 23). The steps to correct the shift are explained below:

1. Choose "View" and then "Data Frame Properties," or click right on "Layers" and select "Properties."

2. Go to the "Coordinate System" tab and click on the plus sign $(+)$ next to the "Layers" folder - all the projections of the layers present in the table of contents will be listed.

3. Select the RLCM layer's coordinate system and click "OK."

The data frame coordinate system is now the same as the RLCM point layer, and the points are aligned (fig. 23).

\section{How do I Change the Classification System?}

Once a class is selected, the user cannot change the classification system (see the "Visualize and Choose a Classification System" section) unless the user is starting a new project and creating a new RLCM layer.

I Have Added a Class but it is Not Showing Up in the "RLCM Attributer" Window or in the RLCM Layer Legend.

Open the "RLCM Classification System" window and make sure the new class is in the "Selected classes" list box (fig. 6). Close and re-open the "RLCM Attributer" window (fig. 9).

\section{How do I Cancel if I Made a Mistake Attributing My Points?}

It is not possible to cancel the attributing of points; however, the last selected points can be reselected using the "Reselect last attributed points" button from the "RLCM Attributer" window (fig. 9), and reattributed to a different class (see the "Overview of the Rapid Land Cover Mapper Attributer" and "Selecting and Attributing Points" sections).

\section{Can I Change the Resolution of My Map After I Have Started Mapping?}

Yes, the user can create a nested grid of a different resolution using the same RLCM layer. Open the "RLCM Layer and Time Periods" window (fig. 4) - the "Study area name" and "Output folder" fields will be automatically populated and must not be changed. Enter a new "Point grid resolution" to create new points at a different resolution, which will be stored as the default resolution in the $\mathrm{xml}$ file.

\section{Can I Have Two Rapid Land Cover Mapper Layers in One ArcMap Project?}

Yes, the RLCM layers are identified by the "_rlcm" text string. Make sure to select the correct RLCM layer in the "RLCM Laer and Time Periods," "RLCM Classification System," and "RLCM Attributer" windows before using any of the tools.

\section{Can I Change the Projection of the Rapid Land Cover Mapper Layer?}

No, the projection of the RLCM layer cannot be changed. The projection is assigned automatically by the RLCM add-in. The data frame of the ArcMap project can be changed, but the RLCM points may appear shifted.

\section{Why is the Tool Slow When Attributing and Selecting Points?}

As the RLCM layer shapefile increases in size, selecting and attributing points may become slow. To avoid this issue, make sure the "ToolConfig $\backslash$ BuildShapeIndex" property in the .xml file is set to "True." This tag is set to "True" by default. When working with smaller datasets $(30,000$ points or less), the user has the option of turning off the "BuildShapeIndex" property, which may improve screen redrawing performance.

\section{Can I Create Dots at a Scale Less Than $1: 800,000$ ?}

Yes, in the .xml file the "ToolConfig $\backslash$ DotCreationScale" property can be changed. Creating dots at a scale less than 1:800,000 may affect the performance of the RLCM add-in and is not advised.

\section{Why Can I Not Create Points Within the Mask?}

Check the projection of your mask if the points do not appear; project your mask to the same projection as your RLCM layer.

\section{Get an Error Message When Trying to Open the "RLCM Attributer" Window. How do I Fix It?}

Move the RLCM layer to the top of the table of contents. The RLCM point layer should always be the first layer in the table of contents. 
Data Frame Properties

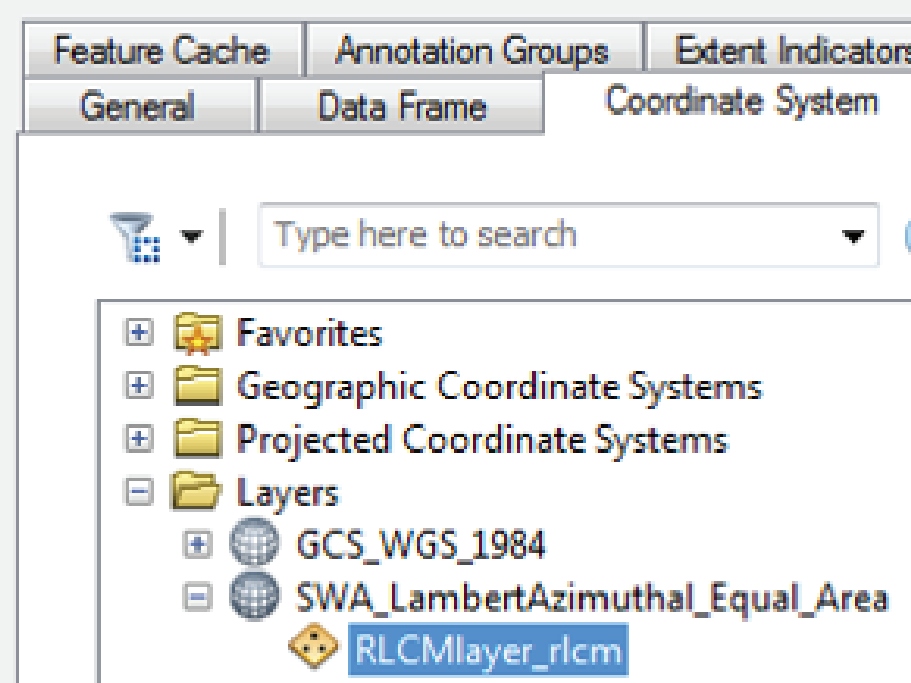

Current coordinate system:

SWA_LambertAzimuthal_Equal_Area

Authority: Custom

Projection: Lambert_Azimuthal_Equal_Area

False_Easting: 0.0

False_Northing: 0.0

Central_Meridian: 3.0

Latitude_of_Origin: 11.0

Linear Unit: $\bar{M}$ eter (1.0)

Geographic Coordinate System: GCS_WGS_1984

Transformations...

\section{OK}

Figure 22. "Coordinate System" tab of the "Data Frame Properties" window. 

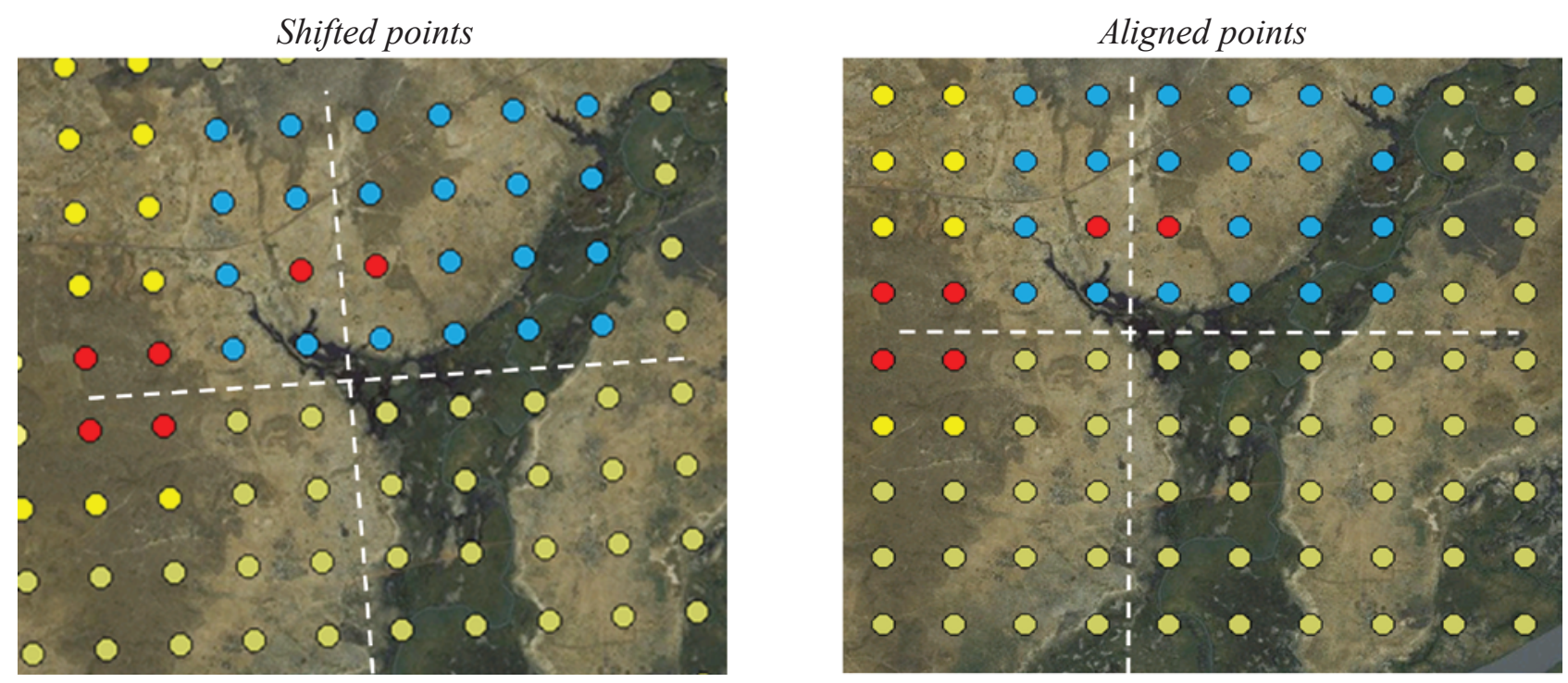

Figure 23. Shifted points (left) and aligned points (right) after updating the data frame projection.

\section{When Using the "Google Earth" Button, Why do the Google Earth Images Appear Zoomed In?}

Change the data frame projection to a meters-based projection or the same as the RLCM layer's projection.

\section{What do I do if the Rapid Land Cover Mapper Add-In Keeps Crashing?}

If the tool and the ArcMap project keep crashing, there are a few things to check:

- Make sure the data frame projection is the same projection as the RLCM layer's projection.

- Check the output working directory for the "[studyareaname]_rlcm.xml" file. If this file does not exist, the tool will not work.

- The RLCM point layer must exist in your table of contents to use the RLCM add-in.

- Make sure your version of ArcMap is 10.1 or greater.

- Check the table of contents for data layers that have broken links. Remove them and save your project.

\section{What do I do if I Get an Error Message When I Try to Create Points?}

If the "Create Points" button (fig. 4) is not working, try the following:

- Make sure the data frame projection uses meters.
- Check the "[studyareaname]_rlcm" working directory and make sure the "[studyareaname]_rlcm.xml" file exists.

- Zoom in to a scale of 1:800,000 or greater and try again.

- Try moving the RLCM points layer to the top of the table of contents.

- Points will not be created if the table of contents is empty. Add imagery or reference datasets to the ArcGIS project and then try to create points.

\section{References Cited}

Comité permanent Inter-états de Lutte contre la Sécheresse dans le Sahel [CILSS], 2016, Landscapes of West AfricaA window on a changing world: Ouagadougou, Burkina Faso, CILSS, 219 p., https://doi.org/10.5066/F7N014QZ.

Lillesand, T.M., Kiefer, R.W., and Chipman, J.W., 2015, Remote sensing and image interpretation (7th ed.): John Wiley \& Sons, $736 \mathrm{p}$.

Schumacher, F.X., and Chapman, R.A., 1942, Sampling methods in forestry and range management: Durham, N.C., Duke University, School of Forestry Bulletin 7, 222 p. 
Appendixes 1-3 


\section{Appendix 1. Classification Systems Included in the Rapid Land Cover Mapper Add-In}

Classification systems embedded in the Rapid Land Cover Mapper are provided in table 1-1 (an Excel file is available for download at https://doi.org/ofr20171012).

\section{Appendix 2. List of the Rapid Land Cover Mapper Projection Files}

The Rapid Land Cover Mapper layer's projected coordinate systems (fig. 2-1) are continental standard projections based on Lambert Azimuthal Equal-Area Projection, with the exception of West Africa that has a unique central meridian and latitude of origin -3 and 11 , respectively.

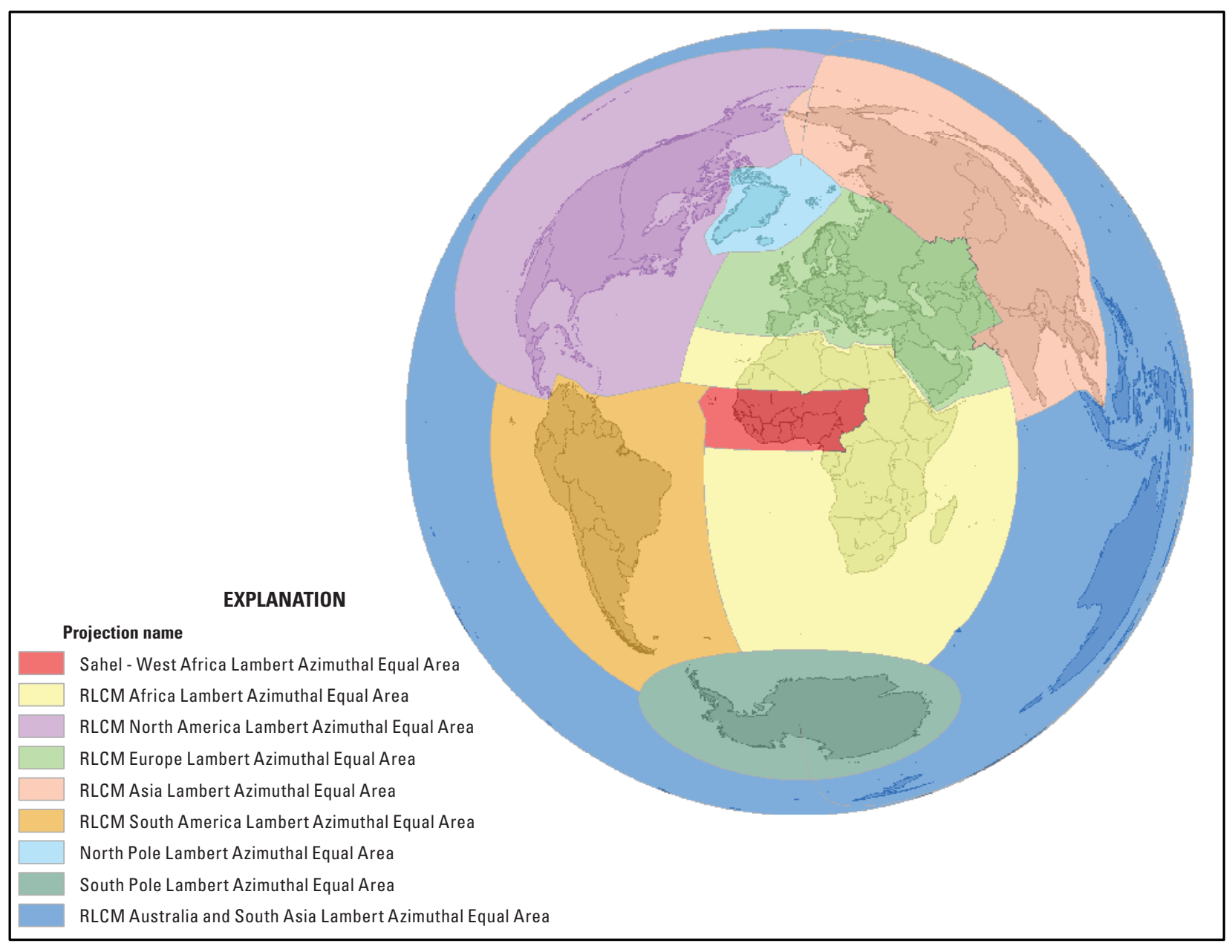

Figure 2-1. Projections embedded in the Rapid Land Cover Mapper add-in. 


\section{Appendix 3. Description of the XML File}

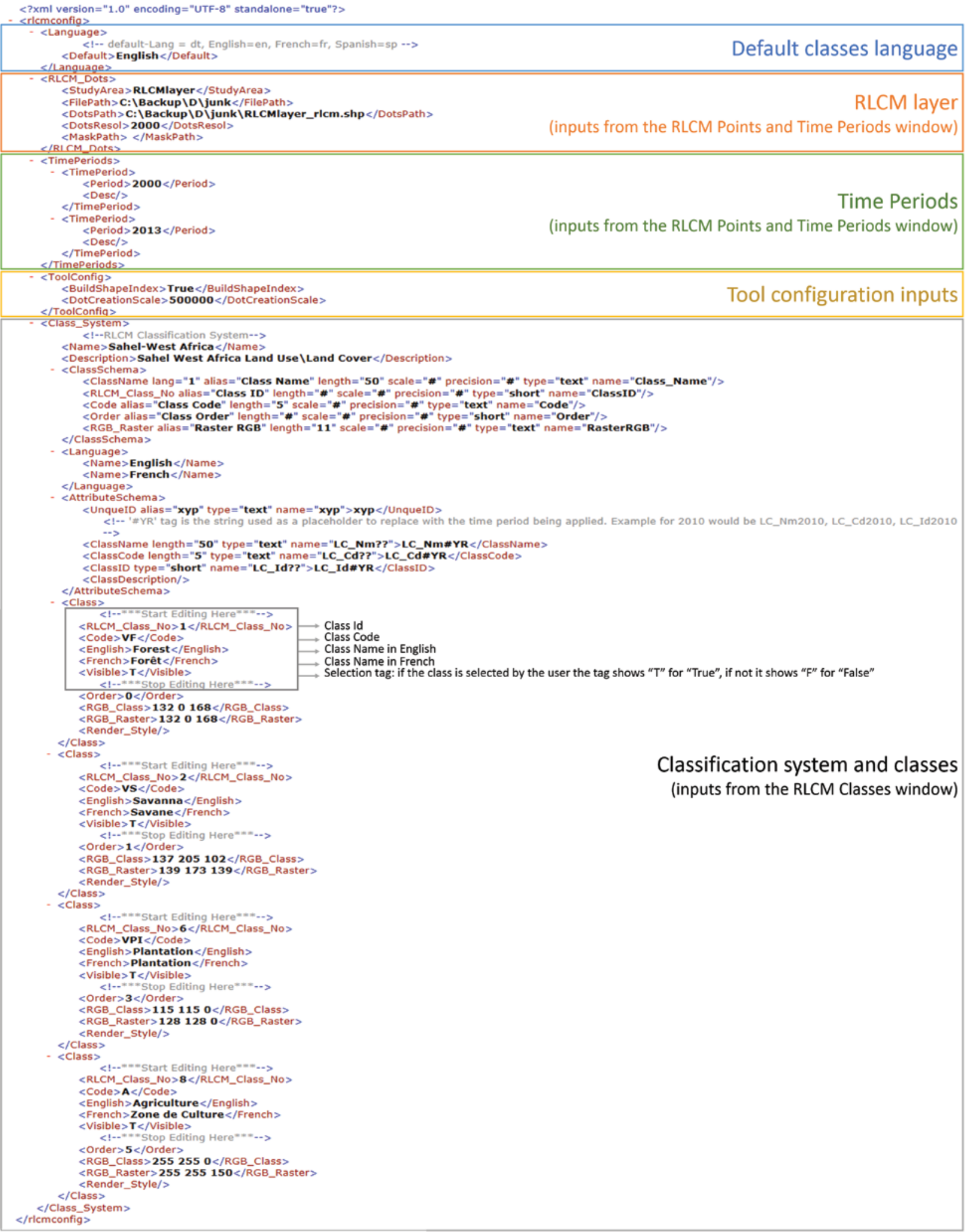

Figure 3-1. The structure of an .xml file from a Rapid Land Cover Mapper layer.Shifted points (left) and aligned points (right) after updating the data frame projection. 
Publishing support provided by: Rolla Publishing Service Center

For additional information concerning this publication, contact:

Director, USGS Earth Resources Observation and Science (EROS) Center

47914 252nd Street

Sioux Falls, SD 57198

(605) 594-6151

Or visit the EROS Center website at: https://www.eros.usgs.gov 



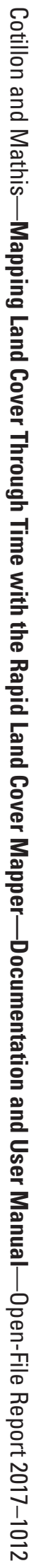

